\title{
COLUMN TESSELLATIONS
}

\author{
NGOC Linh NGUYen ${ }^{\bowtie, 1}$, Viola Weiss ${ }^{2}$ AND Richard COWAN ${ }^{3}$ \\ ${ }^{1}$ Friedrich-Schiller-Universität Jena, Institut für Stochastik, Germany; ${ }^{2}$ Ernst-Abbe-Hochschule Jena, \\ Fachbereich Grundlagenwissenschaften, Germany; ${ }^{3}$ School of Mathematics and Statistics, University of Sydney, \\ Australia \\ e-mail: linh.nguyen@uni-jena.de,viola.weiss@fh-jena.de, richard.cowan@sydney.edu.au \\ (Received February 3, 2015; revised May 19, 2015; accepted May 26, 2015)
}

\begin{abstract}
A new class of non facet-to-facet random tessellations in three-dimensional space is introduced - the so-called column tessellations. The spatial construction is based on a stationary planar tessellation; each cell of the spatial tessellation is a prism whose base facet is congruent to a cell of the planar tessellation. Thus intensities and various mean values of the spatial tessellation can be calculated from suitably chosen parameters of the planar tessellation.
\end{abstract}

Keywords: combinatorial topology, random tessellations, stochastic geometry.

\section{THE CONTEXT FOR OUR NEW MODEL}

Random tessellations are classical structures considered by stochastic geometers. Two standard models are the Poisson hyperplane and Poisson Voronoi tessellations (Schneider and Weil, 2008; Chiu et al., 2013). In the planar case these tessellations are side-to-side, that is, each side of a polygonal tessellation cell coincides with a side of a neighbouring cell. In their three dimensional versions they are facetto-facet, meaning that each facet of a polyhedral cell coincides with a facet of a neighbouring cell.

In recent years there has been a growing interest in tessellation models that do not have the stated coincidence for all sides or facets. A first systematic study of the effects when a three-dimensional tessellation is not facet-to-facet is given in Weiss and Cowan (2011). A recent study presented in Cowan and Thäle (2014) looks in depth at the planar case, building on results from the 1970s when non side-toside tessellations first attracted attention.

Tessellations of that kind arise for example by cell division. Among these models, the iteration stable or STIT tessellation is of particular interest because of the number of analytically available results (Nagel and Weiss, 2005; Mecke et al., 2008; Thäle et al., 2012; Cowan, 2013; Thäle and Weiss, 2013, and the references therein). It serves as a reference model for geological crack and fissure structures (Mosser and Matthäi, 2014) and might have application in the process of biological cell division. The development of new model classes is important for further applications to random structures in materials science, geology and biology - and the current paper contributes to that aim.
We consider a new class of non facet-to-facet spatial tessellations, whose construction is based on a stationary planar tessellation $\mathscr{Y}^{\prime}$ having convex polygonal cells. From each polygonal cell z of $\mathscr{Y}^{\prime}$ we form an infinite column perpendicular to the plane $\mathscr{E}$ in which $\mathscr{Y}^{\prime}$ lies. Any cross-section of the column parallel to $\mathscr{E}$ is congruent to z. To create a spatial tessellation, each infinite column is intersected by many such cross-sections, thereby dividing the column into cells. The spatial cells which arise are prisms and their polygonal base facets (located at the cross-sections) are translations (in the third dimension orthogonal to $\mathscr{E}$ ) of the cells of $\mathscr{Y}^{\prime}$. The resulting three-dimensional tessellation $\mathscr{Y}$ is called a column tessellation. Column tessellations could be useful to describe crack structures in geology, as for example in the Giant's Causeway of Northern Ireland (Figs. 1 and 2).

If the locations of cross-sections were identical in all columns, the column tessellation $\mathscr{Y}$ reduces to the stratum model of (Mecke, 1984) and is facet-to-facet if and only if $\mathscr{Y}^{\prime}$ is side-to-side. So, in order to be innovative, we shall be introducing mechanisms such that no section which divides a column is coplanar with a section of a neighbouring column. This implies that cells in neighbouring columns will not have a common facet. As a consequence, the tessellations we shall study in this paper are never facet-to-facet.

The definitions which we shall use to specify where the cross-sections intersect the columns can vary, thus giving scope for us to consider different cases - and so to construct a rich model class. This class provides a significant generalization of column constructions considered briefly in (Weiss and Cowan, 2011). In the current paper we investigate which 
parameters of the planar tessellation are necessary to calculate characteristics of the spatial tessellation. This is interesting, for example, when only a planar section through a spatial column tessellation can be observed.

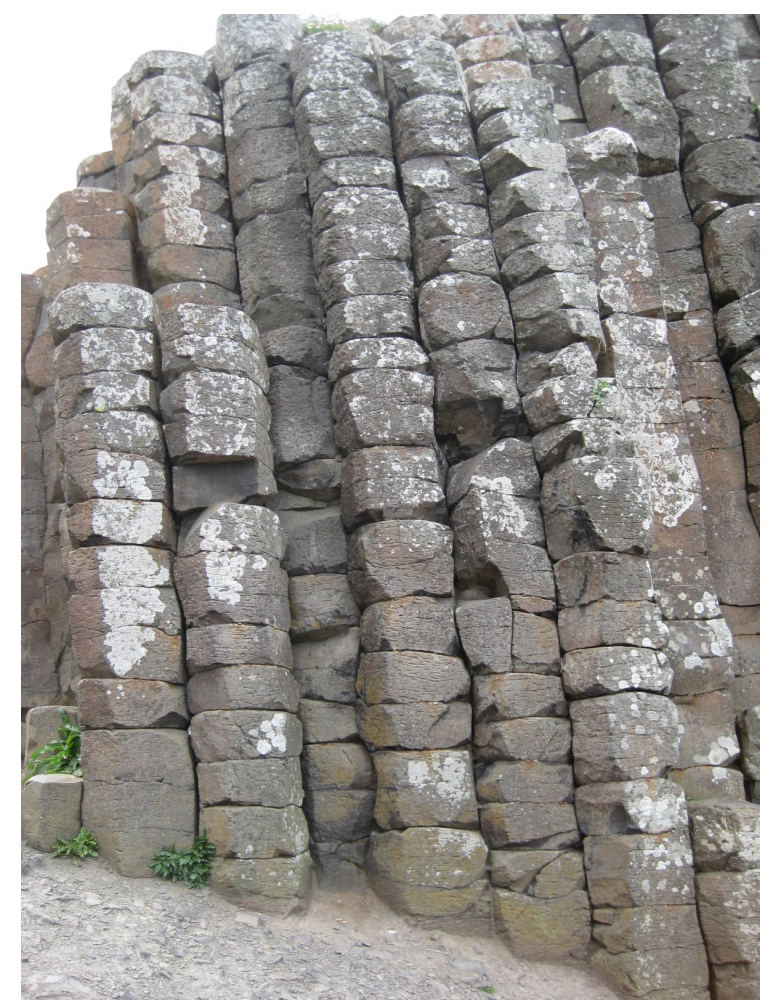

Fig. 1. Basalt columns, approximately $6-8$ metres high above ground level, divided by 'cross-sectional plates' at approximately $30 \mathrm{~cm}$ spacing. Photo taken by one of the authors. There are many formations like this one (at the Giant's Causeway, Northern Ireland) around the world.

The paper is organized as follows. To describe in detail the complicated geometry for tessellations which are not facet-to-facet, we use the system of notation given in (Weiss and Cowan, 2011). The next section gives a short introduction to this notation for planar and spatial tessellations. In Section "Column tessellations", the general construction of a column tessellation is explained, special notation is defined and basic properties are considered. Section "Formulae for the features of column tessellations" shows that intensities and various mean values of a spatial column tessellation can be determined by suitably chosen parameters of the planar tessellation. Later in that section, formulae for metric mean values of the column tessellation are also deduced. Three examples conclude the paper.

To aid comprehension throughout the paper, we often consider the special case where the crosssectional plates in a column have a constant separation of 1 unit. This generates a column tessellation where all the cells have height 1 . We illustrate the notation and our results using this special case.
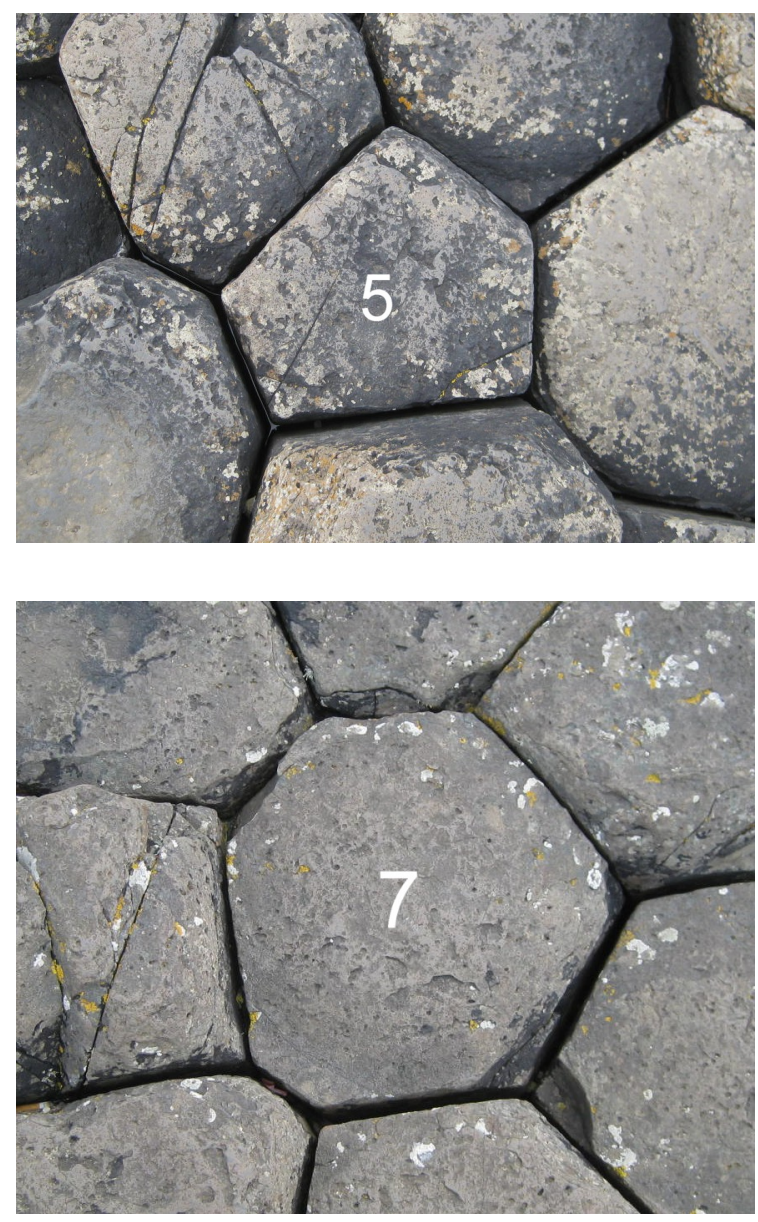

Fig. 2. Viewed from above, examples of pentagonal and heptagonal basalt columns at the Giant's Causeway.

\section{BASIC DEFINITIONS AND THE NOTATION USED}

The geometric elements: In this paper we describe stationary convex-celled random tessellations in $\mathbb{R}^{2}$ and $\mathbb{R}^{3}$ in general terms. We use the structure and system of notation given in Weiss and Cowan (2011), where convexity of cells was also assumed. Convexity implies that the cells in the $\mathbb{R}^{2}$ case are convex polygons and, in the $\mathbb{R}^{3}$ case, convex polyhedra.

In the three-dimensional case, we avoid a clash in terminology between tessellation theory and polyhedra theory by calling the faces of a polyhedron as follows: apex (a 0-face); ridge (a 1-face); facet (a 2-face). The words side and corner describe the 1-faces and 0faces of a polygon - and this terminology will be used both for the cells of an $\mathbb{R}^{2}$-tessellation and for 
polygons imbedded in an $\mathbb{R}^{3}$-tessellation. For example, cell-facets in a tessellation of $\mathbb{R}^{3}$ are polygons; so we can speak of facet-sides and facet-corners.

For a spatial tessellation, we deal with four kinds of primitive elements: these are called vertices, edges, plates and cells. They are defined as follows.

A tessellation of $\mathbb{R}^{3}$ is a locally-finite collection of compact convex cells which cover the threedimensional space and overlap only on their boundaries. The union of the cell boundaries is called the tessellation frame. A subset of the frame, the union of all the cell-ridges, is called the tessellation net. Each cell has apices, ridges and facets which lie on the frame, the first two lying in the net. The union of all apices is a collection of points in $\mathbb{R}^{3}$ called the vertices of the tessellation. Those line-segments which are contained in the net, have a vertex at each end and no vertices in their relative interior are called edges of the tessellation. Those convex polygons which are contained in the frame, whose boundary is contained in the net and whose relative interior is disjoint from the net are called plates.

So we see that vertices, edges, plates and cells are elements of the tessellation, whilst apex, ridge and facet are names for elements of a polyhedral cell. Vertices, edges, plates and cells are primitive in the sense that they cannot have any other elements in their relative interior.

Classes of elements and their inter-relation: The corresponding classes of these primitives are denoted by $V, E, P$ and $Z$. An object belonging to a class $X$ is often referred to as "an X-type object" or "an object of type X".

For a random tessellation the intensity of objects of class $X$ is denoted by $\lambda_{X}$. It is the mean number of centroids of X-type objects per unit volume. By definition, the centroid of a nonempty compact subset $C$ of $\mathbb{R}^{d}$ is the centre of the (uniquely determined) smallest ball containing $C$ (Schneider and Weil, 2008, Section 4.1). It is assumed henceforth that $0<\lambda_{x}<\infty$; this is the case for all examples considered. Recall that an object $\mathrm{X}$ of $\mathrm{X}$ is said to be adjacent to an object $\mathrm{y}$ of $Y$ if either $\mathrm{x} \subseteq \mathrm{y}$ or $\mathrm{y} \subseteq \mathrm{x}$. Let $\mu_{\mathrm{XY}}$ be the mean number of $Y$-type objects adjacent to the typical object of $X$. Formally the typical object of class $X$ can be introduced by means of Palm distributions for which we refer to Schneider and Weil (2008) and Chiu et al. (2013). Intuitively it can be considered as a uniformly selected object from $X$ independently of its size and shape. For an element $x \in X$ the number of $Y$-type objects adjacent to $\mathrm{x}$ is denoted by $m_{\mathrm{Y}}(\mathrm{x})$. Formally, we write $\mu_{\mathrm{XY}}:=\mathbb{E}_{\mathrm{X}}\left[m_{\mathrm{Y}}(\mathrm{x})\right]$, where $\mathbb{E}_{\mathrm{X}}$ denotes an expectation for the typical object of type $X$ with respect to the Palm distribution.

Because of combinatorial and topological relations within any particular spatial tessellation the twelve adjacency mean values $\mu_{X Y}$, for $X, Y \in\{V, E, P, Z\}$ and $X \neq Y$, can be expressed for a random tessellation as functions of just three parameters; they have cyclic subscripts.

$$
\begin{aligned}
& \mu_{\mathrm{VE}}-\quad \text { the mean number of edges emanating } \\
& \text { from the typical vertex, } \\
& \mu_{\mathrm{EP}}-\quad \begin{array}{l}
\text { the mean number of plates emanating } \\
\text { from the typical edge and }
\end{array} \\
& \mu_{\mathrm{PV}}-\quad \begin{array}{l}
\text { the mean number of vertices on the } \\
\text { boundary of the typical plate. }
\end{array}
\end{aligned}
$$

See Weiss and Cowan (2011) or also Radecke (1980) and Mecke (1984) where another notation is used. Note that all twelve adjacencies are invariant under topological transformations of $\mathbb{R}^{3}$, defined in Grünbaum and Shephard (1987, page 166). So we call $\mu_{\mathrm{VE}}, \mu_{\mathrm{EP}}$ and $\mu_{\mathrm{PV}}$ the topological parameters.

In the facet-to-facet case, the $j$-dimensional faces of an X-type object are primitive elements. In contrast, for non facet-to-facet tessellations we must carefully distinguish between the primitive elements and the $j$-faces of polytopes. For example a cell can have vertices on its boundary which are not 0 -faces of that polytope. A 1-face (ridge) of a cell can have vertices in its relative interior, this being impossible for edges. A 2-face (facet) of a cell may not be a plate. Hence we use the notation $X_{j}$ for the class of all $j$-faces of $\mathrm{X}$-type polytopes, $j<\operatorname{dim}(\mathrm{X}$-object $)$. For instance $\mathrm{P}_{1}$ is the class of the 1-dimensional faces of all plates, called the plate-sides. We emphasize that some of these classes are multisets because of the multiplicities of the elements. For example if $k$ cells have a 0 face located at a vertex $v$, then the class $Z_{0}$ has $k$ elements positioned on $\mathrm{v}$; note that here $k \leq m_{\mathrm{Z}}(\mathrm{v})$. Furthermore, we define $n_{j}(\mathrm{x})$ as the number of $j$-faces of a particular object $\mathrm{x} \in \mathrm{X}$ and $v_{j}(\mathrm{X}):=\mathbb{E}_{\mathrm{X}}\left[n_{j}(\mathrm{x})\right]$ is the mean number of $j$-faces of the typical $X$-object. For example it is

$$
\begin{aligned}
& v_{0}(\mathrm{P})-\quad \text { the mean number of } 0 \text {-faces of the } \\
& \text { typical plate, } \\
& v_{1}(\mathrm{Z})-\quad \begin{array}{l}
\text { the mean number of } 1 \text {-faces (ridges) } \\
\text { of the typical cell. }
\end{array}
\end{aligned}
$$

Sometimes we use $X[\cdot]$ for a subset of the class $X$, where the term in the brackets is a suitably chosen symbol describing the property of the subclass. For example, the subclasses of horizontal and vertical edges are denoted by $\mathrm{E}[\mathrm{hor}]$ and $\mathrm{E}$ [vert] respectively.

Parameters when not facet-to-facet: If a spatial tessellation is not facet-to-facet, a face of a primitive 
element can have interior structure. To quantify the effects of this phenomenon four additional parameters for a random tessellation were introduced in Weiss and Cowan (2011). They are called interior parameters and defined as follows:

$$
\begin{aligned}
& \xi \quad-\quad \text { the proportion of edges whose interiors } \\
& \text { are contained in the interior of some } \\
& \text { cell-facet, } \\
& \kappa \quad-\quad \text { the proportion of vertices in the } \\
& \text { tessellation contained in the interior } \\
& \text { of some cell-facet, } \\
& \psi \quad-\quad \text { the mean number of ridge-interiors } \\
& \text { adjacent to the typical vertex, } \\
& \tau \quad-\quad \text { the mean number of plate-side-interiors } \\
& \text { adjacent to the typical vertex. }
\end{aligned}
$$

Note that the interior parameters can be written using the adjacency notation as

$$
\xi=\mu_{\mathrm{EZ}}^{\circ}, \quad \kappa=\mu_{\mathrm{VZ}_{2}}^{\circ}, \quad \psi=\mu_{\mathrm{VZ}_{1}} \text { and } \tau=\mu_{\mathrm{VP}_{1}}^{\circ},
$$

using $\stackrel{\circ}{X}$ for the class of relative interiors of members of $X$. We call an edge whose interior is contained in the interior of a cell-facet a $\pi$-edge and a vertex in the interior of a cell-facet a hemi-vertex (Weiss and Cowan, 2011).

Naturally all four interior parameters are zero in the facet-to-facet case. In Cowan and Weiss (2015) it is shown that a random spatial tessellation is facet-tofacet if and only if $\xi=0$. Some further notation will be given later.

The planar tessellation: The initial template for the construction of a column tessellation is a stationary planar tessellation $\mathscr{Y}^{\prime}$ in a fixed plane $\mathscr{E}$ which, without loss of generality, is assumed horizontal. The classes of planar primitive elements of $\mathscr{Y}^{\prime}$ are $\mathrm{V}^{\prime}$ (vertices), $E^{\prime}$ (edges) and $Z^{\prime}$ (cells); analogously to the spatial case these entities are defined for planar tessellations (Cowan and Thäle, 2014). For example, an edge of a planar tessellation is a line segment which is contained in the frame, has a vertex on each end and no vertices in its relative interior. All the intensities $\lambda_{X}^{\prime}$ and the adjacency mean values $\mu_{X Y}^{\prime}$, $\mathrm{X}, \mathrm{Y} \in\left\{\mathrm{V}^{\prime}, \mathrm{E}^{\prime}, \mathrm{Z}^{\prime}\right\}$ are marked with an apostrophe. The subscripts are always written without apostrophes. A planar tessellation which is not side-to-side has vertices located in the interior of cell-sides. We call them $\pi$-vertices, because one angle created by the emanating edges is equal to $\pi$. The interior parameter of a random planar tessellation is

$$
\begin{gathered}
\phi \quad-\quad \text { the proportion of } \pi \text {-vertices in the } \\
\text { tessellation, } \phi=\mu_{\mathrm{V} Z_{1}}^{\prime} .
\end{gathered}
$$

Two cells of a planar tessellation are called neighbours if their intersection is an edge of the tessellation.

\section{COLUMN TESSELLATIONS}

Construction: Based on the planar tessellation $\mathscr{Y}^{\prime}$ in the horizontal plane $\mathscr{E}$ we construct the spatial column tessellation $\mathscr{Y}$ in the following way:

For each cell $z$ of $\mathscr{Y}^{\prime}$, we consider an infinite cylindrical column based on this cell and perpendicular to $\mathscr{E}$. Further we mark z's centroid with a real-valued positive $\rho_{\mathrm{z}}$. Here, conditional upon $\mathscr{Y}^{\prime}, \rho_{\mathrm{z}}$ is a nonrandom function of some aspects of $\mathscr{Y}^{\prime}$ viewed from $z$, for example, the size, shape or environment of the cell z. (Unconditionally, $\rho_{z}$ inherits some randomness from $\mathscr{Y}^{\prime}$.) Such a mark is created for all cells in $\mathscr{Y}^{\prime}$. Now, for each planar cell z, we construct on the line going through the cell-centroid of z perpendicular to $\mathscr{E}$ - we call this the cell-axis - a stationary and simple point process with intensity $\rho_{\mathrm{z}}$. This point process may be dependent on the cell-axis point processes for neighbouring columns (the columns based on two neighbouring cells of $\mathscr{Y}^{\prime}$ ), but we require the following property.

Property 3.1. The simple superposition property: We require that the superposition of the cell-axis point processes of two neighbouring columns be a simple point process, that is, a point process without multiplicities.

To create the spatial tessellation, the column based on $z$ is intersected by horizontal cross-sections located at each of the random points of that column's point process. The resulting tessellation $\mathscr{Y}$ is called a column tessellation. Note that the cell-axes do not belong to the column tessellation. Because the horizontal cross-sections are actually plates of $\mathscr{Y}$, and there are no other plates in the tessellation that are horizontal, we shall often refer to them as the horizontal plates.

Any cell of $\mathscr{Y}$ is a right prism, where its base facet is a vertical translation of a cell of $\mathscr{Y}^{\prime}$. Because of Property 3.1 , there is no coplanarity of cross-sectional plates that appear in neighbouring columns, and so the cells in neighbouring columns cannot have a common facet. So a column tessellation is not facet-to-facet. The intersection of a column tessellation $\mathscr{Y}$ with any plane parallel to $\mathscr{E}$ is a vertical translation of $\mathscr{Y}^{\prime}$.

Constant cell heights: Let $\zeta_{k}, k=0, \pm 1, \pm 2, \ldots$, be the random distances of the horizontal plates from $\mathscr{E}$. A very simple case arises when $\zeta_{0}$ is uniformly distributed in $(0,1]$ and $\zeta_{k}=\zeta_{0}+k$ for all $k$. This 
implies, of course, that $\rho_{z}=1$, a constant for all cells $z$ of $\mathscr{Y}^{\prime}$. Also the positions of the horizontal plates in a column are stationary and completely independent of such plates in the neighbouring columns, as no information has been drawn from $\mathscr{Y}^{\prime}$.
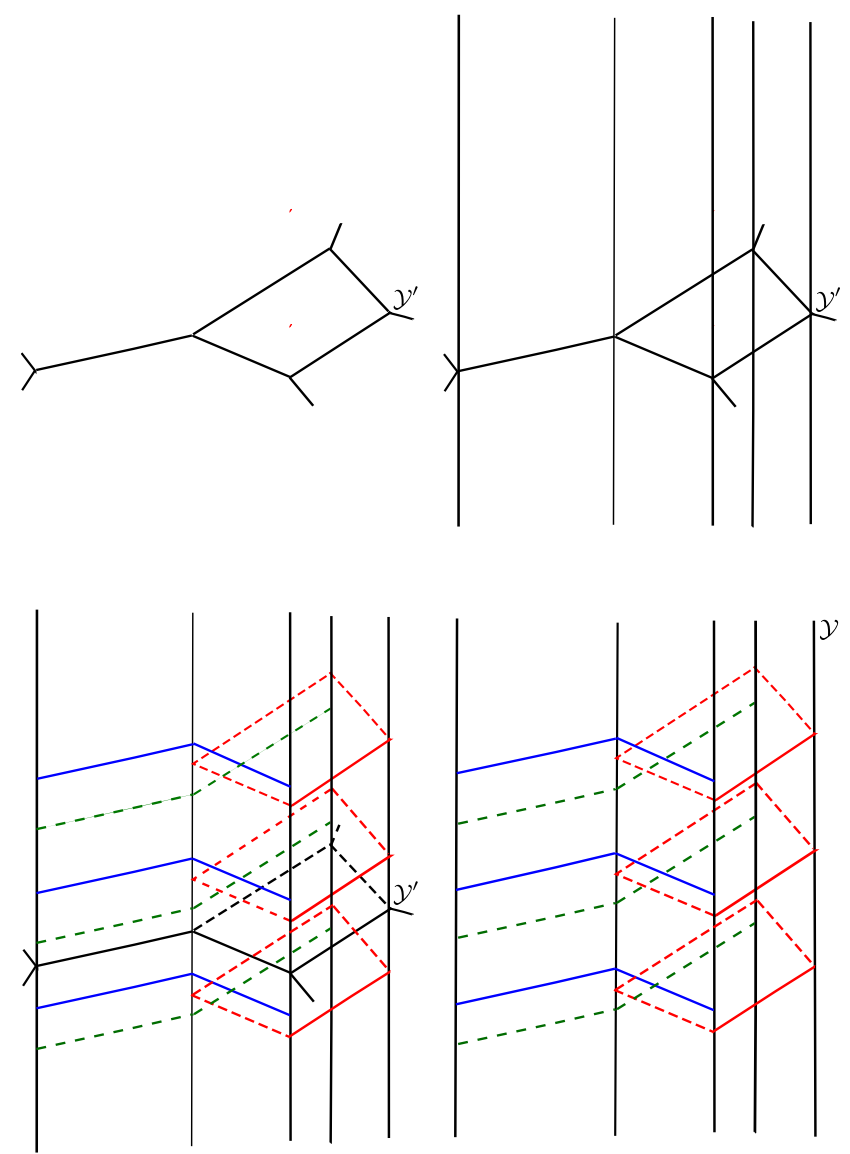

Fig. 3. Column tessellation $\mathscr{Y}$ with constant height 1. Here the four steps building up a column tessellation are shown: Starting with the planar tessellation $\mathscr{Y}^{\prime}$, next the columns are given by the cells of $\mathscr{Y}^{\prime}$. Then the columns with their cuts are shown, whereas in the last figure $\mathscr{Y}^{\prime}$ is removed, it is no part of $\mathscr{Y}$.

Any cell of the column tessellation $\mathscr{Y}$ that has arisen is a right prism with height 1 . For short, we call it a column tessellation with height 1 . An illustration is given in Fig. 3. On the top on the left the planar tessellation $\mathscr{Y}^{\prime}$ is shown and the columns formed by the cells of $\mathscr{Y}^{\prime}$ on the right. On the bottom left we see the columns with the cuts generated by the parallel horizontal plates, using three different colors for three columns. Down the right we strike $\mathscr{Y}^{\prime}$ off because it is not a part of the column tessellation $\mathscr{Y}$.

More notation: We need further notation for planar tessellations. Some is based on a relationship between cells and lower dimensional objects of the planar tessellation - an ownership relation. We describe the ownership relation using a function $b$ (belonging to) defined as follows. If $\mathrm{z}_{j}$ is a $j$-face of a cell $z, j=0,1$, then it belongs to that cell and we write $\mathrm{z}=b\left(\mathrm{z}_{j}\right)$ to identify the owner. In other words, cell $\mathrm{z}$ is the owner of any $j$-face $\mathrm{z}_{j}$ such that $\mathrm{z}=b\left(\mathrm{z}_{j}\right)$. It is obvious that $\mathrm{z}$ is the owner of $n_{j}(\mathrm{z}) j$-faces and that any $z_{j} \in Z_{j}^{\prime}$ has its unique owner. (Remember that $Z_{j}^{\prime}$ is a multiset.) Furthermore we are interested in the vertices of a cell which are not corners ( 0 -faces) of that cell. It is obvious that those vertices are $\pi$-vertices. We say that $z$ is the owner of such a $\pi$-vertex which is not a 0 -face of $z$. We use for this special belonging to function the symbol $b_{\pi}$. Thus any $\pi$-vertex $\mathrm{v}[\pi]$ belongs to a unique owner-cell $\mathrm{z}=b_{\pi}(\mathrm{v}[\pi])$ and a cell $\mathrm{z}$ owns $m_{\mathrm{V}}(\mathrm{z})-n_{0}(\mathrm{z}) \pi$-vertices. So our belonging to function $b$ has domain $Z_{0}{ }^{\prime} \cup Z_{1}{ }^{\prime}$ and range $Z^{\prime}$, whereas function $b_{\pi}$ has domain $\mathrm{V}^{\prime}[\pi]$ and range $\mathrm{Z}^{\prime}$.

Later we will investigate the dependency of the vertex intensity of a column tessellation on both the planar tessellation and the given marks $\rho_{\mathrm{z}}$. It is easy to see that all vertices of $\mathscr{Y}$ are located on vertical lines through the vertices of $\mathscr{Y}^{\prime}$. Also the intensity of the vertices on such a vertex line depends on the $\rho$ intensity of all the planar cells adjacent to the planar vertex which creates this vertex line. To describe those relations between $\mathscr{Y}, \mathscr{Y}^{\prime}$ and the cell marks $\rho_{\mathrm{z}}$ we define further entities for the planar tessellation $\mathscr{Y}^{\prime}$ as follows.

- When $x$ is adjacent to $z \in Z^{\prime}$,

$$
\alpha_{x}:=\sum_{\{z: z \supset x\}} \rho_{z}
$$

where we mostly consider the cases $x=v \in V^{\prime}$, $\mathrm{x}=\mathrm{e} \in \mathrm{E}^{\prime}$ and $\mathrm{x}=\mathrm{v}[\pi] \in \mathrm{V}^{\prime}[\pi]$.

- When $z \in Z^{\prime}$ owns $z_{0} \in Z_{0}^{\prime}$ or $v[\pi] \in \mathrm{V}^{\prime}[\pi]$,

$$
\begin{aligned}
\beta_{\mathrm{z}_{0}} & :=\rho_{b\left(\mathrm{z}_{0}\right)} \quad \text { and } \\
\beta_{\mathrm{v}[\pi]} & :=\rho_{b_{\pi}(\mathrm{v}[\pi])}
\end{aligned}
$$

where $\mathrm{z}_{0}$ is a 0 -face of the cell $b\left(\mathrm{z}_{0}\right)$ and $\mathrm{v}[\pi]$ is a $\pi$-vertex and no 0 -face of the cell $b_{\pi}(\mathrm{v}[\pi])$.

Furthermore,

- if $\ell^{\prime}(\mathrm{e})$ is the length of the edge e and $a^{\prime}(\mathrm{z})$ is the area of the cell $z$,

$$
\begin{array}{ll}
\gamma_{\mathrm{v}}:=m_{\mathrm{E}}^{\prime}(\mathrm{v}) \alpha_{\mathrm{v}} & \text { (number-weighted), } \\
\gamma_{\mathrm{e}}:=\ell^{\prime}(\mathrm{e}) \alpha_{\mathrm{e}} & \text { (length-weighted), } \\
\gamma_{\mathrm{z}}:=a^{\prime}(\mathrm{z}) \rho_{\mathrm{z}} & \text { (area-weighted). }
\end{array}
$$




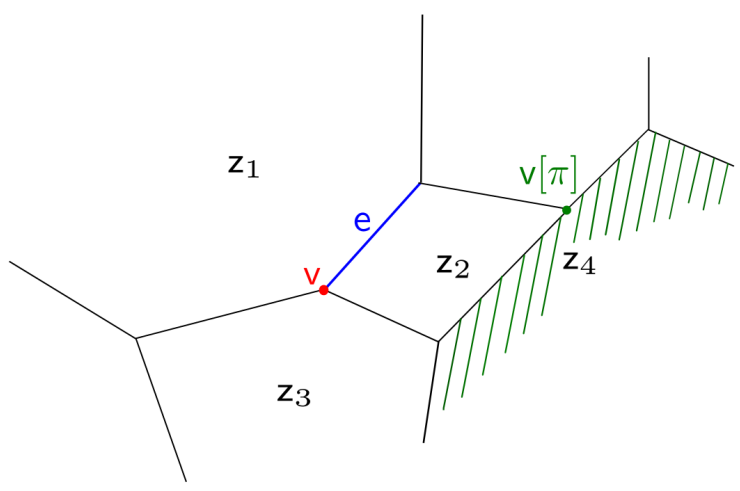

Fig. 4. An example of adjacency and ownership relations in the planar tessellation $\mathscr{Y}^{\prime}$.

Fig. 4 illustrates an example that shows our notation - and also the differences between the ownership and adjacency relations. The vertex $v$ is adjacent to the cells $z_{1}, z_{2}$ and $z_{3}$, the edge e is adjacent to the cells $z_{1}$ and $z_{2}$, hence $\alpha_{v}=\rho_{z_{1}}+\rho_{z_{2}}+\rho_{z_{3}}$ and $\alpha_{\mathrm{e}}=\rho_{\mathrm{z}_{1}}+\rho_{\mathrm{z}_{2}}$. For the ownership relation, it is easy to see that for the $\pi$-vertex $v[\pi]$ we have $\beta_{\mathrm{v}[\pi]}=\rho_{\mathrm{z}_{4}}$, because $\mathrm{z}_{4}=b_{\pi}(\mathrm{v}[\pi])$. Besides, $\mathrm{v}$ is a 0 -face of the cells $z_{1}, z_{2}$ and $z_{3}$, then the class $z_{0}^{\prime}$ has 3 elements located on $v$ denoted by $z_{01}, z_{02}$ and $z_{03}$ (not shown in the figure) with owner-cells $z_{1}, z_{2}$ and $z_{3}$, respectively. Hence $\beta_{\mathrm{z}_{01}}=\rho_{b\left(\mathrm{z}_{01}\right)}=\rho_{\mathrm{z}_{1}}, \beta_{\mathrm{z}_{02}}=\rho_{\mathrm{z}_{2}}$ and $\beta_{\mathrm{z}_{03}}=\rho_{\mathrm{z}_{3}}$.

Later we will see that the last two items of the new notation, $\gamma_{\mathrm{e}}$ and $\gamma_{\mathrm{z}}$, are necessary for the studies of the metrical properties whereas all others are used for results concerning topological and interior parameters. All these $\alpha$-, $\beta$-, $\gamma$-quantities can be understood as marks of elements of the planar tessellation. Each of these marks leads to mark distributions. The corresponding means in the random context are as follows:

- $\quad \bar{\rho}_{Z}=\mathbb{E}_{Z}^{\prime}\left(\rho_{Z}\right)$ - the mean $\rho$-intensity of the typical cell;

- $\quad \bar{\alpha}_{\mathrm{X}}=\mathbb{E}_{\mathrm{X}}^{\prime}\left(\alpha_{\mathrm{x}}\right)$ - the mean total $\rho$-intensity of all cells adjacent to the typical $X$-object, $X \in$ $\left\{\mathrm{V}^{\prime}, \mathrm{V}^{\prime}[\pi], \mathrm{E}^{\prime}\right\}$

- $\bar{\beta}_{\mathrm{Z}_{0}}=\mathbb{E}_{\mathrm{Z}_{0}}^{\prime}\left(\beta_{\mathrm{z}_{0}}\right)$ and $\bar{\beta}_{\mathrm{V}[\pi]}=\mathbb{E}_{\mathrm{V}[\pi]}^{\prime}\left(\beta_{\mathrm{v}[\pi]}\right)$ - the mean $\rho$-intensity of the owner cell of the typical 0 -face or the typical $\pi$-vertex, respectively;

- $\quad \bar{\gamma}_{X}=\mathbb{E}_{X}^{\prime}\left(\gamma_{X}\right)$ - the mean total weighted $\rho$-intensity of all cells adjacent to the typical $X$-object, $X \in$ $\left\{V^{\prime}, E^{\prime}, Z^{\prime}\right\}$.

Remark 3.2. Using mean value identities for the primitive tessellation elements, given in (Møller, 1989), and some generalisations, most of the above mean values can be expressed as second-order quantities depending on the $\rho$-intensity as follows:

- $\lambda_{X}^{\prime} \bar{\alpha}_{X}=\lambda_{Z}^{\prime} \mathbb{E}_{Z}^{\prime}\left(m_{X}^{\prime}(z) \rho_{z}\right)$;

- $\lambda_{\mathrm{Z}_{0}}^{\prime} \bar{\beta}_{\mathrm{Z}_{0}}=\lambda_{\mathrm{Z}}^{\prime} \mathbb{E}_{\mathrm{Z}}^{\prime}\left(n_{0}^{\prime}(\mathrm{z}) \rho_{\mathrm{z}}\right)$;

- $\lambda_{\mathrm{V}[\pi]}^{\prime} \bar{\beta}_{\mathrm{V}[\pi]}=\lambda_{\mathrm{Z}}^{\prime} \mathbb{E}_{\mathrm{Z}}^{\prime}\left[\left(m_{\mathrm{V}}^{\prime}(\mathrm{z})-n_{0}^{\prime}(\mathrm{z})\right) \rho_{\mathrm{z}}\right]$

$$
=\lambda_{\mathrm{V}}^{\prime} \bar{\alpha}_{\mathrm{V}}-\lambda_{\mathrm{Z}_{0}}^{\prime} \bar{\beta}_{\mathrm{Z}_{0}}
$$

- $\lambda_{\mathrm{E}}^{\prime} \bar{\gamma}_{\mathrm{E}}=\lambda_{\mathrm{Z}}^{\prime} \mathbb{E}_{\mathrm{Z}}^{\prime}\left(\ell^{\prime}(\mathrm{z}) \rho_{\mathrm{z}}\right)$, where $\ell^{\prime}(\mathrm{z})$ is the perimeter of the planar cell $\mathrm{z}$;

- $\lambda_{\mathrm{Z}}^{\prime} \bar{\gamma}_{\mathrm{Z}}=\lambda_{\mathrm{Z}}^{\prime} \mathbb{E}_{\mathrm{Z}}^{\prime}\left(a^{\prime}(\mathrm{z}) \rho_{\mathrm{Z}}\right)$.

Only $\overline{\mathcal{X}}$ requires a separate argument:

$$
\begin{aligned}
\lambda_{\mathrm{V}}^{\prime} \bar{\gamma} & =\lambda_{\mathrm{Z}}^{\prime} \mathbb{E}_{\mathrm{Z}}^{\prime}\left[\left(k_{\mathrm{E}}^{\prime}(\mathrm{z})+m_{\mathrm{V}}^{\prime}(\mathrm{z})\right) \rho_{\mathrm{z}}\right] \\
& =\lambda_{\mathrm{Z}}^{\prime} \mathbb{E}_{\mathrm{Z}}^{\prime}\left(k_{\mathrm{E}}^{\prime}(\mathrm{z}) \rho_{\mathrm{z}}\right)+\lambda_{\mathrm{V}}^{\prime} \bar{\alpha}_{\mathrm{V}},
\end{aligned}
$$

where $k_{\mathrm{E}}^{\prime}(\mathrm{z}):=\sum_{\mathrm{e} \in \mathrm{E}^{\prime}} \mathbf{1}\{\mathrm{e} \cap \mathrm{z} \neq \emptyset\}$ - the number of edges intersecting z. Here $\mathbf{1}\{\cdot\}$ is the indicator function.

Remark 3.3. To illustrate these mean values we consider now the special case when $\rho_{\mathrm{z}}=1$ for all $\mathrm{z} \in \mathbf{Z}^{\prime}$.

$$
\begin{aligned}
\bar{\rho}_{\mathrm{Z}} & =1, \\
\bar{\alpha}_{\mathrm{V}} & =\mu_{\mathrm{VZ}}^{\prime}=\mu_{\mathrm{VE}}^{\prime}, \\
\bar{\alpha}_{\mathrm{E}} & =2, \\
\bar{\alpha}_{\mathrm{V}[\pi]} & =\mu_{\mathrm{V}[\pi] \mathrm{Z}}^{\prime}=\mu_{\mathrm{V}[\pi] \mathrm{E}}^{\prime}, \\
\bar{\beta}_{\mathrm{Z}_{0}} & =1, \\
\bar{\beta}_{\mathrm{V}[\pi]} & =1, \\
\bar{\gamma}_{\mathrm{V}} & =\mu_{\mathrm{V} \mathrm{Z}}^{\prime(2)}=\mu_{\mathrm{VE}}^{\prime(2)}, \\
\bar{\gamma}_{\mathrm{E}} & =2 \bar{\ell}_{\mathrm{E}}^{\prime}, \\
\bar{\gamma}_{\mathrm{Z}} & =\bar{a}_{\mathrm{Z}}^{\prime},
\end{aligned}
$$

where

$$
\begin{array}{ll}
\mu_{\mathrm{V}[\pi] \mathrm{E}}^{\prime} & -\quad \begin{array}{l}
\text { the mean number of emanating } \\
\text { edges from the typical } \pi \text {-vertex, }
\end{array} \\
\mu_{\mathrm{VE}}^{\prime(2)} & -\quad \begin{array}{l}
\text { the second moment of the } \\
\text { number of edges adjacent to the } \\
\end{array} \\
& \text { typical vertex, } \\
\bar{\ell}_{\mathrm{E}}^{\prime} & -\quad \text { the mean length of the typical edge, } \\
\bar{a}_{\mathrm{Z}}^{\prime} & -\quad \text { the mean area of the typical cell. }
\end{array}
$$

Formally, the second moment of the number of edges adjacent to the typical vertex is given by $\mathbb{E}_{\mathrm{V}}^{\prime}\left[m_{\mathrm{E}}^{\prime}(\mathrm{v})^{2}\right]$, where $\mathbb{E}_{\mathrm{V}}^{\prime}$ denotes an expectation for the typical vertex of $\mathscr{Y}^{\prime}$ with respect to the Palm measure (Chiu et al., 2013).

The first and the last of the formulae in Remark 3.3 are obvious. The formulae for the three $\alpha$-means follow from $\alpha_{\mathrm{x}}=m_{\mathrm{Z}}^{\prime}(\mathrm{x})$ in the case $\rho_{\mathrm{z}}=1$. The $\beta$-mean value formulae arise from the fact that the owner-cell of the typical corner or the typical $\pi$-vertex, respectively, 
has $\rho$-intensity 1 . And the first two $\gamma$-mean values we obtain using again $\alpha_{\mathrm{x}}=m_{\mathrm{Z}}^{\prime}(\mathrm{x})$ for $\mathrm{x}=\mathrm{v}$ and $\mathrm{x}=\mathrm{e}$.

Considering again the general construction, our aim is the calculation of intensities and mean values of the column tessellation $\mathscr{Y}$ from the characteristics of $\mathscr{Y}^{\prime}$. For this purpose the following basic interrelationship between vertices and edges of $\mathscr{Y}$ and $\mathscr{Y}^{\prime}$ is helpful.

Basic properties: For a vertex $\vee \in \mathrm{V}^{\prime}$ in $\mathscr{Y}^{\prime}$ we consider the vertical line $\mathscr{L}_{\mathrm{v}}$ through $\vee$ (called vertexline) and its intersection with the columns created by the planar cells adjacent to $\mathrm{v}$. The horizontal plates in these columns create a point process (comprising vertices of the spatial tessellation $\mathscr{Y}$ ) on $\mathscr{L}_{\mathrm{v}}$, this point process being the superposition of point processes with $\rho$-intensities from the planar cells adjacent to v. Hence it has intensity $\alpha_{v}$. For short we say that $v$ has $\alpha_{v}$ corresponding vertices in $\mathscr{Y}$.

Property 3.4. Let $\mathrm{v}$ be a vertex in $\mathscr{Y}^{\prime}$. Then $\mathrm{v}$ has $\alpha_{\mathrm{v}}$ corresponding vertices in $\mathscr{Y}$ and each one is adjacent to $m_{\mathrm{E}}^{\prime}(\mathrm{v})+1$ cells and to $m_{\mathrm{E}}^{\prime}(\mathrm{v})+3$ plates of $\mathscr{Y}$.

Furthermore the column tessellation has only horizontal and vertical edges denoted by $\mathrm{E}[\mathrm{hor}]$ and $\mathrm{E}$ [vert], respectively. All horizontal edges are $\pi$-edges with three emanating plates. For each edge e of $\mathscr{Y}^{\prime}$, we have two planar cells adjacent to this edge. When we cut the two corresponding columns by different horizontal planes, the intensity of horizontal edges of $\mathscr{Y}$ in the common face of the two neighbouring columns is $\alpha_{\mathrm{e}}$, and all these edges are translations of e. Besides, the intensity of vertical edges of $\mathscr{Y}$ on a vertex line $\mathscr{L}_{\mathrm{v}}$ is $\alpha_{\mathrm{v}}$.

Property 3.5. An edge e of $\mathscr{Y}^{\prime}$ corresponds to $\alpha_{\mathrm{e}}$ horizontal edges of $\mathscr{Y}$. Any horizontal edge of $\mathscr{Y}$ is $a \pi$-edge with three emanating plates, two of them are vertical, the third one is a horizontal plate. A vertex $\mathrm{v}$ of $\mathscr{Y}^{\prime}$ corresponds to $\alpha_{v}$ vertical edges of $\mathscr{Y}$, where each one is adjacent to $m_{\mathrm{E}}^{\prime}(\mathrm{v})$ plates of $\mathscr{Y}$.

These correspondence relations between $\mathscr{Y}^{\prime}$ and $\mathscr{Y}$ will be refined later in the paper.

\section{FORMULAE FOR THE FEATURES OF COLUMN TESSELLATIONS}

Intensities of primitive elements: As a first step we will consider how the intensities $\lambda_{X}$ of the primitive elements $\mathrm{X} \in\{\mathrm{V}, \mathrm{E}, \mathrm{P}, \mathrm{Z}\}$ of a column tessellation $\mathscr{Y}$ depend on characteristics of the planar tessellation $\mathscr{Y}^{\prime}$. To establish formulae for this dependence we need the intensities $\lambda_{Z}^{\prime}$ and $\lambda_{V}^{\prime}$, the mean $\rho$-intensity $\bar{\rho}_{Z}$ and the mean total $\rho$-intensity $\bar{\alpha}_{\vee}$ of $\mathscr{Y}^{\prime}$ :
Proposition 4.1. The intensities of primitive elements of a column tessellation $\mathscr{Y}$ depend on $\mathscr{Y}^{\prime}$ and the cell marks $\rho_{\mathrm{z}}$ as follows:

(i) $\lambda_{V}=\lambda_{V}^{\prime} \bar{\alpha}_{V}$;

(ii) $\lambda_{\mathrm{E}}=2 \lambda_{\mathrm{V}}^{\prime} \bar{\alpha}_{\mathrm{V}}$;

(iii) $\lambda_{\mathrm{P}}=\lambda_{\mathrm{V}}^{\prime} \bar{\alpha}_{\mathrm{V}}+\lambda_{\mathrm{Z}}^{\prime} \bar{\rho}_{\mathrm{Z}}$

(iv) $\lambda_{\mathrm{Z}}=\lambda_{\mathrm{Z}}^{\prime} \bar{\rho}_{\mathrm{Z}}$.

For a refined partition of the classes $\mathrm{E}$ and $\mathrm{P}$ of $\mathscr{Y}$ into horizontal and vertical elements we obtain

(v) $\lambda_{\mathrm{E}[\mathrm{hor}]}=\lambda_{\mathrm{E}[\mathrm{vert}]}=\lambda_{\mathrm{V}}^{\prime} \bar{\alpha}_{\mathrm{V}}$ and

(vi) $\lambda_{\mathrm{P}[\text { hor }]}=\lambda_{\mathrm{Z}}^{\prime} \bar{\rho}_{\mathrm{Z}}, \lambda_{\mathrm{P}[\text { vert }]}=\lambda_{\mathrm{V}}^{\prime} \bar{\alpha}_{\mathrm{V}}$.

Proof. With Property 3.4 we obtain (i).

Using Property 3.5 and the mean value identity $\lambda_{\mathrm{V}}^{\prime} \bar{\alpha}_{\mathrm{V}}=\lambda_{\mathrm{Z}}^{\prime} \mathbb{E}_{\mathrm{Z}}^{\prime}\left(m_{\mathrm{V}}^{\prime}(\mathrm{z}) \rho_{\mathrm{z}}\right)=\lambda_{\mathrm{Z}}^{\prime} \mathbb{E}_{\mathrm{Z}}^{\prime}\left(m_{\mathrm{E}}^{\prime}(\mathrm{z}) \rho_{\mathrm{z}}\right)=\lambda_{\mathrm{E}}^{\prime} \bar{\alpha}_{\mathrm{E}}$ we have (v) and $\lambda_{\mathrm{E}}=\lambda_{\mathrm{E}[\mathrm{hor}]}+\lambda_{\mathrm{E}[\mathrm{vert}]}$ yields (ii).

From the construction of the column tessellation (iv) is obvious. With $\lambda_{V}-\lambda_{E}+\lambda_{P}-\lambda_{Z}=0$ we obtain (iii) and $\lambda_{\mathrm{P}[\text { hor }]}=\lambda_{\mathrm{Z}}$ leads to (vi).

Further intensities can be calculated using properties of the column tessellation or formulae given in Theorem 4.2 and in Weiss and Cowan (2011), for example,

$$
\begin{aligned}
\text { intensity of plate-sides: } & \lambda_{\mathrm{P}_{1}}=\lambda_{\mathrm{Z}_{0}}^{\prime} \bar{\beta}_{\mathrm{Z}_{0}}+4 \lambda_{\mathrm{V}}^{\prime} \bar{\alpha}_{\mathrm{V}} \\
\text { intensity of cell-facets: } & \lambda_{\mathrm{Z}_{2}}=2 \lambda_{\mathrm{Z}}^{\prime} \bar{\rho}_{\mathrm{Z}}+\lambda_{\mathrm{Z}_{0}}^{\prime} \bar{\beta}_{\mathrm{Z}_{0}}, \\
\text { intensity of cell-ridges: } & \lambda_{\mathrm{Z}_{1}}=3 \lambda_{\mathrm{Z}_{0}}^{\prime} \bar{\beta}_{\mathrm{Z}_{0}} .
\end{aligned}
$$

Note that in the calculation of those intensities, the interior parameter $\phi$ of the planar tessellation is a necessary input, because $\lambda_{Z_{0}}^{\prime}$ depends on $\phi$. Namely $\lambda_{\mathrm{Z}_{0}}^{\prime}=\lambda_{\mathrm{V}}^{\prime}\left(\mu_{\mathrm{VE}}^{\prime}-\phi\right)$ (Weiss and Cowan, 2011, Table 3a).

Formulae for the topological and interior parameters: We now present the three adjacency parameters $\mu_{\mathrm{VE}}, \mu_{\mathrm{EP}}, \mu_{\mathrm{PV}}$ and the four interior parameters $\xi, \kappa, \psi, \tau$ of a column tessellation. To clarify their dependence on the basic planar tessellation $\mathscr{Y}^{\prime}$, we need from $\mathscr{Y}^{\prime}$ the mean number of emanating edges of the typical vertex $\mu_{\mathrm{VE}}^{\prime}$, the interior parameter $\phi$ and five already-mentioned mean values $\bar{\rho}_{\mathrm{Z}}, \bar{\alpha}_{\mathrm{V}}, \bar{\alpha}_{\mathrm{V}[\pi]}, \bar{\beta}_{\mathrm{Z}_{0}}, \bar{\gamma}_{\mathrm{V}}$. 
Theorem 4.2. The three topological and four interior parameters of a column tessellation $\mathscr{Y}$ are given by seven parameters of the underlying planar tessellation $\mathscr{Y}^{\prime}$ and the function $\rho_{\mathrm{z}}$ as follows

$$
\begin{aligned}
\mu_{\mathrm{VE}} & =4, \\
\mu_{\mathrm{PV}} & =\frac{2\left(3 \bar{\alpha}_{\mathrm{V}}+\bar{\gamma}_{\mathrm{V}}\right)}{2 \bar{\alpha}_{\mathrm{V}}+\left(\mu_{\mathrm{VE}}^{\prime}-2\right) \bar{\rho}_{\mathrm{Z}}}, \\
\mu_{\mathrm{EP}} & =\frac{1}{2} \frac{\bar{\gamma}_{\bar{\alpha}}}{\bar{\alpha}_{\mathrm{V}}}+\frac{3}{2}, \\
\xi & =\frac{1}{2} \phi \frac{\bar{\alpha}_{\mathrm{V}[\pi]}}{\bar{\alpha}_{\mathrm{V}}}+\frac{1}{2}, \\
\kappa & =\phi \frac{\bar{\alpha}_{\mathrm{V}[\pi]}}{\bar{\alpha}_{\mathrm{V}}}+\left(\mu_{\mathrm{VE}}^{\prime}-\phi\right) \frac{\bar{\beta}_{\mathrm{Z}_{0}}}{\bar{\alpha}_{\mathrm{V}}}-1, \\
\psi & =\frac{\bar{\gamma}_{V}}{\bar{\alpha}_{\mathrm{V}}}-\phi \frac{\bar{\alpha}_{\mathrm{V}[\pi]}}{\bar{\alpha}_{\mathrm{V}}}-3\left(\mu_{\mathrm{VE}}^{\prime}-\phi\right) \frac{\bar{\beta}_{\mathrm{Z}_{0}}}{\bar{\alpha}_{\mathrm{V}}}+2, \\
\tau & =\frac{\bar{\gamma}_{\mathrm{\alpha}}}{\bar{\alpha}_{\mathrm{V}}}-\left(\mu_{\mathrm{VE}}^{\prime}-\phi\right) \frac{\bar{\beta}_{\mathrm{Z}_{0}}}{\bar{\alpha}_{\mathrm{V}}}-1 .
\end{aligned}
$$

Note that the Greek letters overset with a bar are derived from $\rho_{\mathrm{z}}$ and $\mathscr{Y}^{\prime}$.

Proof. 1. Each vertex of $\mathscr{Y}$ arises from the intersection of an infinite cylindrical column with a horizontal plane, hence the vertex has 4 outgoing edges; 2 of them are horizontal and the other 2 are vertical and collinear. So we have $m_{\mathrm{E}}(\mathrm{v})=4$ for all $v \in \mathrm{V}$.

2. From Property 3.4 we have for the mean number of plates adjacent to the typical vertex

$$
\lambda_{\mathrm{V}} \mu_{\mathrm{VP}}=\lambda_{\mathrm{V}}^{\prime} \mathbb{E}_{\mathrm{V}}^{\prime}\left[\alpha_{\mathrm{v}}\left(m_{\mathrm{E}}^{\prime}(\mathrm{v})+3\right)\right]=\lambda_{\mathrm{V}}^{\prime} \bar{\gamma}+3 \lambda_{\mathrm{V}}^{\prime} \bar{\alpha}_{\mathrm{V}} .
$$

With $\lambda_{V} \mu_{\mathrm{VP}}=\lambda_{\mathrm{P}} \mu_{\mathrm{PV}}$ and (iii) of Proposition 4.1 we obtain (2).

3. A column tessellation has horizontal and vertical plates.

$$
\lambda_{\mathrm{E}} \mu_{\mathrm{EP}}=\lambda_{\mathrm{E}[\text { hor }} \mu_{\mathrm{E}[\text { hor }] \mathrm{P}}+\lambda_{\mathrm{E}[\text { vert }]} \mu_{\mathrm{E}[\text { vert }] \mathrm{P}} .
$$

Obviously, $\mu_{\mathrm{E}[\text { hor }] \mathrm{P}}=3$ and we have $\lambda_{\mathrm{E}[\text { hor }]}=\lambda_{V}^{\prime} \bar{\alpha}_{V}$ from (v). Each vertical edge corresponding to a vertex $v$ in the planar tessellation is adjacent to $m_{\mathrm{E}}^{\prime}(\mathrm{v})$ plates; see Property 3.5. Therefore

$$
\lambda_{\mathrm{E}[\text { vert }]} \mu_{\mathrm{E}[\text { vert }] \mathrm{P}}=\lambda_{\mathrm{V}}^{\prime} \mathbb{E}_{\mathrm{V}}^{\prime}\left(\alpha_{\mathrm{v}} m_{\mathrm{E}}^{\prime}(\mathrm{v})\right)=\lambda_{\mathrm{V}}^{\prime} \overline{\mathcal{X}},
$$

and hence, with (ii) of Proposition 4.1,

$$
\mu_{\mathrm{EP}}=\frac{\lambda_{V}^{\prime} \bar{\alpha}_{V} \cdot 3+\lambda_{V}^{\prime} \bar{\psi}_{V}}{2 \lambda_{V}^{\prime} \bar{\alpha}_{V}}=\frac{1}{2} \frac{\bar{\psi}_{V}}{\bar{\alpha}_{V}}+\frac{3}{2} .
$$

4. To find the formulae for the interior parameters we have to refine the correspondence relations between
$\mathscr{Y}^{\prime}$ and $\mathscr{Y}$ into two cases: whether a vertex of $\mathscr{Y}^{\prime}$ is a $\pi$-vertex or not. To calculate the intensity of $\pi$-edges $\lambda_{\mathrm{E}[\pi]}$ of $\mathscr{Y}$ we note firstly that all horizontal edges are $\pi$-edges and secondly that a vertical edge is a $\pi$-edge if the corresponding vertex $v \in \mathscr{Y}^{\prime}$ is a $\pi$-vertex. Hence

$$
\begin{aligned}
\lambda_{\mathrm{E}[\pi]} & =\lambda_{\mathrm{E}[\text { hor }]}+\lambda_{\mathrm{V}[\pi]}^{\prime} \mathbb{E}_{\mathrm{V}[\pi]}^{\prime}\left(\alpha_{\mathrm{V}[\pi]}\right) \\
& =\lambda_{\mathrm{V}}^{\prime} \bar{\alpha}_{\mathrm{V}}+\lambda_{\mathrm{V}[\pi]}^{\prime} \bar{\alpha}_{\mathrm{V}[\pi]},
\end{aligned}
$$

which implies, using $\lambda_{\mathrm{E}[\pi]}=\lambda_{\mathrm{E}} \xi$ and $\lambda_{\mathrm{E}}=2 \lambda_{V}^{\prime} \bar{\alpha}_{\mathrm{V}}$, that

$$
\xi=\frac{\lambda_{\mathrm{V}}^{\prime} \bar{\alpha}_{\mathrm{V}}+\lambda_{\mathrm{V}[\pi]}^{\prime} \bar{\alpha}_{\mathrm{V}[\pi]}}{2 \lambda_{\mathrm{V}}^{\prime} \bar{\alpha}_{\mathrm{V}}}=\frac{1}{2} \phi \frac{\bar{\alpha}_{\mathrm{V}[\pi]}}{\bar{\alpha}_{\mathrm{V}}}+\frac{1}{2}
$$

5. To prove Eq. 5 (and Eqs. 6-7 too) we again have to refine the correspondence between $\mathscr{Y}^{\prime}$ and $\mathscr{Y}$. We consider when the vertices of a column tessellation are hemi-vertices or not. If the vertex $v$ of $\mathscr{Y}^{\prime}$ is not a $\pi$-vertex, then all $\alpha_{v}$ corresponding vertices of $\mathscr{Y}$ are not hemi-vertices. If the vertex is a $\pi$-vertex, denoted by $v[\pi]$, then $\beta_{\mathrm{v}[\pi]}$ of the corresponding vertices are non-hemi-vertices, the others hemi-vertices. Hence the intensity of hemi-vertices $\lambda_{V[\kappa]}=\lambda_{V} \kappa$ is

$$
\begin{aligned}
\lambda_{\mathrm{V}[\kappa]} & =\lambda_{\mathrm{V}[\pi]}^{\prime} \mathbb{E}_{\mathrm{V}[\pi]}^{\prime}\left(\alpha_{\mathrm{V}[\pi]}-\beta_{\mathrm{V}[\pi]}\right) \\
& =\lambda_{\mathrm{V}[\pi]}^{\prime} \bar{\alpha}_{\mathrm{V}[\pi]}-\lambda_{\mathrm{V}[\pi]}^{\prime} \bar{\beta}_{\mathrm{V}[\pi]} \\
& =\lambda_{\mathrm{V}[\pi]}^{\prime} \bar{\alpha}_{\mathrm{V}[\pi]}-\lambda_{\mathrm{V}}^{\prime} \bar{\alpha}_{\mathrm{V}}+\lambda_{\mathrm{Z}_{0}}^{\prime} \bar{\beta}_{\mathrm{Z}_{0}}
\end{aligned}
$$

using $\lambda_{\mathrm{V}[\pi]}^{\prime} \bar{\beta}_{\mathrm{V}[\pi]}=\lambda_{\mathrm{V}}^{\prime} \bar{\alpha}_{V}-\lambda_{\mathrm{Z}_{0}}^{\prime} \bar{\beta}_{\mathrm{Z}_{0}}$ from Remark 3.2. Therefore with $\lambda_{\mathrm{Z}_{0}}^{\prime}=\lambda_{\mathrm{V}}^{\prime}\left(\mu_{\mathrm{VE}}^{\prime}-\phi\right)$ and Proposition 4.1(i),

$$
\begin{aligned}
\kappa & =\frac{\lambda_{\mathrm{V}[\pi]}^{\prime} \bar{\alpha}_{\mathrm{V}[\pi]}-\lambda_{\mathrm{V}}^{\prime} \bar{\alpha}_{\mathrm{V}}+\lambda_{\mathrm{V}}^{\prime}\left(\mu_{\mathrm{VE}}^{\prime}-\phi\right) \bar{\beta}_{\mathrm{Z}_{0}}}{\lambda_{\mathrm{V}}^{\prime} \bar{\alpha}_{\mathrm{V}}} \\
& =\phi \frac{\bar{\alpha}_{\mathrm{V}[\pi]}}{\bar{\alpha}_{\mathrm{V}}}+\left(\mu_{\mathrm{VE}}^{\prime}-\phi\right) \frac{\bar{\beta}_{\mathrm{Z}_{0}}}{\bar{\alpha}_{\mathrm{V}}}-1 .
\end{aligned}
$$

6. To present the parameter $\psi$, we have to find out the number of ridge-interiors adjacent to a vertex in different cases. If the vertex $\vee$ of $\mathscr{Y}^{\prime}$ is not a $\pi$-vertex, denoted by $\mathrm{v}[\bar{\pi}]$, then each of the $\alpha_{\mathrm{v}[\bar{\pi}]}$ corresponding vertices of $\mathscr{Y}$ is adjacent to $m_{\mathrm{E}}^{\prime}(v[\bar{\pi}])-1$ ridgeinteriors. If $v$ of $\mathscr{Y}^{\prime}$ is a $\pi$-vertex $v[\pi]$, each of the corresponding non-hemi-vertices of $\mathscr{Y}$ is adjacent to $m_{\mathrm{E}}^{\prime}(\mathrm{v}[\pi])+1$ ridge-interiors, and each of the remaining corresponding hemi-vertices is adjacent to $m_{\mathrm{E}}^{\prime}(\mathrm{v}[\pi])-$ 2 ridge-interiors. Hence 


$$
\begin{aligned}
\lambda_{\mathrm{V} \psi}= & \lambda_{\mathrm{V}[\bar{\pi}]}^{\prime} \mathbb{E}_{\mathrm{V}[\bar{\pi}]}^{\prime}\left[\alpha_{\mathrm{v}[\bar{\pi}]}\left(m_{\mathrm{E}}^{\prime}(\mathrm{v}[\bar{\pi}])-1\right)\right]+ \\
& +\lambda_{\mathrm{V}[\pi]}^{\prime} \mathbb{E}_{\mathrm{V}[\pi]}^{\prime}\left[\beta_{\mathrm{v}[\pi]}\left(m_{\mathrm{E}}^{\prime}(\mathrm{v}[\pi])+1\right)\right]+ \\
& +\lambda_{\mathrm{V}[\pi]}^{\prime} \mathbb{E}_{\mathrm{V}[\pi]}^{\prime}\left[\left(\alpha_{\mathrm{v}[\pi]}-\beta_{\mathrm{v}[\pi]}\right)\left(m_{\mathrm{E}}^{\prime}(\mathrm{v}[\pi])-2\right)\right] \\
= & \lambda_{\mathrm{V}}^{\prime} \mathbb{E}_{\mathrm{V}}^{\prime}\left(\alpha_{\mathrm{v}} m_{\mathrm{E}}^{\prime}(\mathrm{v})\right)-2 \lambda_{\mathrm{V}}^{\prime} \mathbb{E}_{\mathrm{V}}^{\prime}\left(\alpha_{\mathrm{v}}\right)+ \\
& +\lambda_{\mathrm{V}[\bar{\pi}]}^{\prime} \mathbb{E}_{\mathrm{V}[\bar{\pi}]}^{\prime}\left(\alpha_{\mathrm{v}[\bar{\pi}]}\right)+3 \lambda_{\mathrm{V}[\pi]}^{\prime} \mathbb{E}_{\mathrm{V}[\pi]}^{\prime}\left(\beta_{\mathrm{v}[\pi]}\right) \\
= & \lambda_{\mathrm{V}}^{\prime} \overline{\mathcal{X}}_{\mathrm{V}}-2 \lambda_{\mathrm{V}}^{\prime} \bar{\alpha}_{\mathrm{V}}+\lambda_{\mathrm{V}}^{\prime} \bar{\alpha}_{\mathrm{V}}-\lambda_{\mathrm{V}[\pi]}^{\prime} \bar{\alpha}_{\mathrm{V}[\pi]}+ \\
& +3 \lambda_{\mathrm{V}}^{\prime} \bar{\alpha}_{\mathrm{V}}-3 \lambda_{\mathrm{Z}_{0}}^{\prime} \bar{\beta}_{\mathrm{Z}_{0}} \\
= & \lambda_{\mathrm{V}}^{\prime} \bar{\gamma}_{\mathrm{V}}-\lambda_{\mathrm{V}[\pi]}^{\prime} \bar{\alpha}_{\mathrm{V}[\pi]}-3 \lambda_{\mathrm{Z}_{0}}^{\prime} \bar{\beta}_{\mathrm{Z}_{0}}+2 \lambda_{\mathrm{V}}^{\prime} \bar{\alpha}_{\mathrm{V}} .
\end{aligned}
$$

Therefore,

$$
\begin{aligned}
\psi & =\frac{\lambda_{\mathrm{V}}^{\prime} \overline{\mathcal{\gamma}}-\lambda_{\mathrm{V}[\pi]}^{\prime} \bar{\alpha}_{\mathrm{V}[\pi]}-3 \lambda_{\mathrm{V}}^{\prime}\left(\mu_{\mathrm{VE}}^{\prime}-\phi\right) \bar{\beta}_{\mathrm{Z}_{0}}+2 \lambda_{\mathrm{V}}^{\prime} \bar{\alpha}_{\mathrm{V}}}{\lambda_{\mathrm{V}}^{\prime} \bar{\alpha}_{\mathrm{V}}} \\
& =\frac{\bar{\gamma}_{\mathrm{\alpha}}}{\bar{\alpha}_{\mathrm{V}}}-\phi \frac{\bar{\alpha}_{\mathrm{V}[\pi]}}{\bar{\alpha}_{\mathrm{V}}}-3\left(\mu_{\mathrm{VE}}^{\prime}-\phi\right) \frac{\bar{\beta}_{\mathrm{Z}_{0}}}{\bar{\alpha}_{\mathrm{V}}}+2 .
\end{aligned}
$$

7. For this last identity we consider how the number of plate-side-interiors adjacent to a vertex of $\mathscr{Y}$ depends on the type of the corresponding vertex of $\mathscr{Y}^{\prime}$. If the vertex $\vee$ of $\mathscr{Y}^{\prime}$ is a $\vee[\bar{\pi}]$, then each of the corresponding $\alpha_{\mathrm{v}[\bar{\pi}]}$ vertices of $\mathscr{Y}$ is adjacent to $m_{\mathrm{E}}^{\prime}(\mathrm{v}[\bar{\pi}])-2$ plate-side-interiors. If $\vee$ is a $\mathrm{v}[\pi]$, each of the corresponding non-hemi-vertices is adjacent to $m_{\mathrm{E}}^{\prime}(\mathrm{v}[\pi])-1$ plate-side-interiors, and each of the other corresponding hemi-vertices is adjacent to $m_{\mathrm{E}}^{\prime}(\mathrm{v}[\pi])-$ 2 plate-side-interiors. Hence

$$
\begin{aligned}
\lambda_{\mathrm{V} \tau} \tau= & \lambda_{\mathrm{V}[\bar{\pi}}^{\prime} \mathbb{E}_{\mathrm{V}[\bar{\pi}]}^{\prime}\left[\alpha_{\mathrm{v}[\bar{\pi}]}\left(m_{\mathrm{E}}^{\prime}(\mathrm{v}[\bar{\pi}])-2\right)\right]+ \\
& +\lambda_{\mathrm{V}[\pi]}^{\prime} \mathbb{E}_{\mathrm{V}[\pi]}^{\prime}\left[\beta_{\mathrm{v}[\pi]}\left(m_{\mathrm{E}}^{\prime}(\mathrm{v}[\pi])-1\right)\right]+ \\
& +\lambda_{\mathrm{V}[\pi]}^{\prime} \mathbb{E}_{\mathrm{V}[\pi]}^{\prime}\left[\left(\alpha_{\mathrm{v}[\pi]}-\beta_{\mathrm{v}[\pi]}\right)\left(m_{\mathrm{E}}^{\prime}(\mathrm{v}[\pi])-2\right)\right] \\
= & \lambda_{\mathrm{V}}^{\prime} \mathbb{E}_{\mathrm{V}}^{\prime}\left(\alpha_{\mathrm{v}} m_{\mathrm{E}}^{\prime}(\mathrm{v})\right)-2 \lambda_{\mathrm{V}}^{\prime} \mathbb{E}_{\mathrm{V}}^{\prime}\left(\alpha_{\mathrm{v}}\right)+ \\
& +\lambda_{\mathrm{V}[\pi]}^{\prime} \mathbb{E}_{\mathrm{V}[\pi]}^{\prime}\left(\beta_{\mathrm{v}[\pi]}\right) \\
= & \lambda_{\mathrm{V}}^{\prime} \bar{\alpha}-2 \lambda_{\mathrm{V}}^{\prime} \bar{\alpha}_{\mathrm{V}}+\lambda_{\mathrm{V}}^{\prime} \bar{\alpha}_{\mathrm{V}}-\lambda_{\mathrm{Z}_{0}}^{\prime} \bar{\beta}_{\mathrm{Z}_{0}} \\
= & \lambda_{\mathrm{V}}^{\prime} \bar{\gamma}-\lambda_{\mathrm{V}}^{\prime} \bar{\alpha}_{\mathrm{V}}-\lambda_{\mathrm{Z}_{0}}^{\prime} \bar{\beta}_{\mathrm{Z}_{0}} .
\end{aligned}
$$

Therefore

$$
\begin{aligned}
\tau & =\frac{\lambda_{V}^{\prime} \overline{\mathcal{X}}_{V}-\lambda_{\mathrm{V}}^{\prime}\left(\mu_{\mathrm{VE}}^{\prime}-\phi\right) \bar{\beta}_{\mathrm{Z}_{0}}-\lambda_{\mathrm{V}}^{\prime} \bar{\alpha}_{\mathrm{V}}}{\lambda_{\mathrm{V}}^{\prime} \bar{\alpha}_{V}} \\
& =\frac{\bar{\gamma}_{V}}{\bar{\alpha}_{V}}-\left(\mu_{\mathrm{VE}}^{\prime}-\phi\right) \frac{\bar{\beta}_{\mathrm{Z}_{0}}}{\bar{\alpha}_{\mathrm{V}}}-1 .
\end{aligned}
$$

Using identities in Weiss and Cowan (2011), further mean values can be computed. For example the mean number of vertices and edges, respectively, of the typical cell are

$$
\mu_{\mathrm{ZV}}=2 \frac{\bar{\gamma}_{\mathrm{V}}+\bar{\alpha}_{\mathrm{V}}}{\left(\mu_{\mathrm{VE}}^{\prime}-2\right) \bar{\rho}_{\mathrm{Z}}} \quad \text { and } \quad \mu_{\mathrm{ZE}}=2 \frac{\bar{\gamma}_{\mathrm{V}}+3 \bar{\alpha}_{\mathrm{V}}}{\left(\mu_{\mathrm{VE}}^{\prime}-2\right) \bar{\rho}_{\mathrm{Z}}}
$$

whereas the mean number of 0 -faces and 1 -faces of the typical cell are

$$
v_{0}(\mathrm{Z})=\frac{4\left(\mu_{\mathrm{VE}}^{\prime}-\phi\right)}{\left(\mu_{\mathrm{VE}}^{\prime}-2\right)} \frac{\bar{\beta}_{\mathrm{Z}_{0}}}{\bar{\rho}_{\mathrm{Z}}}
$$

and

$$
v_{1}(\mathrm{Z})=\frac{6\left(\mu_{\mathrm{VE}}^{\prime}-\phi\right)}{\left(\mu_{\mathrm{VE}}^{\prime}-2\right)} \frac{\bar{\beta}_{\mathrm{Z}_{0}}}{\bar{\rho}_{\mathrm{Z}}}
$$

Remark 4.3. To calculate the intensities and topological/interior parameters of a column tessellation with height 1 from the planar tessellation, five planar parameters are needed,

$$
\lambda_{\mathrm{V}}^{\prime}, \quad \mu_{\mathrm{VE}}^{\prime}, \quad \phi, \quad \mu_{\mathrm{EV}[\pi]}^{\prime} \quad \text { and } \quad \mu_{\mathrm{VE}}^{\prime(2)}
$$

Using Remark 3.3, Proposition 4.1 and Theorem 4.2 and the mean value identities

$$
\mu_{\mathrm{V}[\pi] \mathrm{E}}^{\prime}=\frac{\mu_{\mathrm{VE}}^{\prime}}{2 \phi} \mu_{\mathrm{EV}[\pi]}^{\prime} \quad \text { and } \quad \lambda_{\mathrm{Z}}^{\prime}=\frac{1}{2} \lambda_{\mathrm{V}}^{\prime}\left(\mu_{\mathrm{VE}}^{\prime}-2\right),
$$

the intensities of a column tessellation with height 1 are

$$
\begin{array}{ll}
\lambda_{\mathrm{V}}=\lambda_{\mathrm{V}}^{\prime} \mu_{\mathrm{VE}}^{\prime}, & \lambda_{\mathrm{E}}=2 \lambda_{\mathrm{V}}^{\prime} \mu_{\mathrm{VE}}^{\prime}, \\
\lambda_{\mathrm{P}}=\frac{1}{2} \lambda_{\mathrm{V}}^{\prime}\left(3 \mu_{\mathrm{VE}}^{\prime}-2\right), & \lambda_{\mathrm{Z}}=\frac{1}{2} \lambda_{\mathrm{V}}^{\prime}\left(\mu_{\mathrm{VE}}^{\prime}-2\right),
\end{array}
$$

the topological parameters are

$$
\begin{aligned}
& \mu_{\mathrm{VE}}=4, \quad \mu_{\mathrm{PV}}=\frac{2}{3 \mu_{\mathrm{VE}}^{\prime}-2}\left(3 \mu_{\mathrm{VE}}^{\prime}+\mu_{\mathrm{VE}}^{\prime(2)}\right), \\
& \mu_{\mathrm{EP}}=\frac{1}{2 \mu_{\mathrm{VE}}^{\prime}}\left(3 \mu_{\mathrm{VE}}^{\prime}+\mu_{\mathrm{VE}}^{\prime(2)}\right),
\end{aligned}
$$

and for the interior parameters we obtain

$$
\begin{array}{ll}
\xi=\frac{1}{2}+\frac{1}{4} \mu_{\mathrm{EV}[\pi]}^{\prime}, & \kappa=\frac{1}{2} \mu_{\mathrm{EV}[\pi]}^{\prime}-\frac{\phi}{\mu_{\mathrm{VE}}^{\prime}}, \\
\psi=\frac{\mu_{\mathrm{VE}}^{\prime(2)}+3 \phi}{\mu_{\mathrm{VE}}^{\prime}}-1-\frac{1}{2} \mu_{\mathrm{EV}[\pi]}^{\prime}, & \tau=\frac{\mu_{\mathrm{VE}}^{\prime(2)}+\phi}{\mu_{\mathrm{VE}}^{\prime}}-2 .
\end{array}
$$

Remark 4.4. In Cowan and Weiss (2015) constraints on the topological/interior parameters of spatial tessellations are considered. They showed that the second moment $\mu_{\mathrm{VE}}^{\prime(2)}$ of a planar tessellation is unbounded. So, in the class of column tessellations with height 1 , the mean values $\mu_{\mathrm{EP}}, \mu_{\mathrm{PV}}$ and $\tau, \psi$ are unbounded. Further constraints follow in Proposition 4.5. 
Proposition 4.5. The constraints for the topological/interior parameters of a column tessellation $\mathscr{Y}$ with height 1 depending on $\mu_{\mathrm{VE}}^{\prime}$ and $\phi$ of $\mathscr{Y}^{\prime}$ are as follows

$$
\begin{aligned}
\frac{36}{7} & \leq \frac{2 \mu_{\mathrm{VE}}^{\prime}\left(3+\mu_{\mathrm{VE}}^{\prime}\right)}{3 \mu_{\mathrm{VE}}^{\prime}-2} \leq \mu_{\mathrm{PV}} \\
3 & \leq \frac{1}{2}\left(3+\mu_{\mathrm{VE}}^{\prime}\right) \leq \mu_{\mathrm{EP}} \\
\frac{1}{2} & \leq \frac{1}{2}+\frac{3}{2} \frac{\phi}{\mu_{\mathrm{VE}}^{\prime}} \leq \xi \leq 1-\frac{3(1-\phi)}{2 \mu_{\mathrm{VE}}^{\prime}} \leq 1, \\
0 & \leq \frac{2 \phi}{\mu_{\mathrm{VE}}^{\prime}} \leq \kappa \leq 1-\frac{3-2 \phi}{\mu_{\mathrm{VE}}^{\prime}} \leq \frac{3}{4}, \\
2 & \leq \mu_{\mathrm{VE}}^{\prime}+\frac{3}{\mu_{\mathrm{VE}}^{\prime}}-2 \leq \psi \\
1 & \leq \mu_{\mathrm{VE}}^{\prime}+\frac{\phi}{\mu_{\mathrm{VE}}^{\prime}}-2 \leq \tau .
\end{aligned}
$$

Proof. For any planar tessellation we have

$$
0 \leq \phi \leq 1 \quad \text { and } \quad 3 \leq \mu_{\mathrm{VE}}^{\prime} \leq 6-2 \phi,
$$

as shown in Weiss and Cowan (2011). Furthermore it is evident that $3 \leq \mu_{\mathrm{V}[\pi] \mathrm{E}}^{\prime}$ and $3 \leq \mu_{\mathrm{V}[\bar{\pi}] \mathrm{E}}^{\prime}$. With help from $\mu_{\mathrm{VE}}^{\prime}=\phi \mu_{\mathrm{V}[\pi] \mathrm{E}}^{\prime}+(1-\phi) \mu_{\mathrm{V}[\bar{\pi}] \mathrm{E}}^{\prime}$ we obtain the following constraints for the mean number of emanating edges of the typical $\pi$-vertex

$$
3 \leq \mu_{\mathrm{V}[\pi] \mathrm{E}}^{\prime} \leq \frac{\mu_{\mathrm{VE}}^{\prime}}{\phi}-\frac{3(1-\phi)}{\phi} .
$$

Hence the constraints for $\mu_{\mathrm{EV}[\pi]}^{\prime}$ are

$$
\frac{6 \phi}{\mu_{\mathrm{VE}}^{\prime}} \leq \mu_{\mathrm{EV}[\pi]}^{\prime} \leq 2-\frac{6(1-\phi)}{\mu_{\mathrm{VE}}^{\prime}}
$$

using $\mu_{\mathrm{EV}[\pi]}^{\prime}=2 \phi \mu_{\mathrm{V}[\pi] \mathrm{E}}^{\prime} / \mu_{\mathrm{VE}}^{\prime}$.

Applying these results together with the inequality $\mu_{\mathrm{VE}}^{\prime(2)} \geq\left(\mu_{\mathrm{VE}}^{\prime}\right)^{2}$ to Remark 4.3 leads to the constraints for column tessellations with height 1 .

Formulae involving the length metric: Firstly we consider mean values corresponding to the length measure for the object classes $X \in\{E, P, Z\}$ in $\mathscr{Y}$, those denoted by

\section{$\bar{\ell}_{X} \quad-\quad$ the mean total length of all 1-faces of the} typical $\mathrm{X}$-object, where $\operatorname{dim}(\mathrm{X}$-object $) \geq 1$.

This yields, for special object classes,

$\bar{\ell}_{E}-$ the mean length of the typical edge,

$\bar{\ell}_{\mathrm{P}}$ - the mean perimeter of the typical plate,

$\bar{\ell}_{Z} \quad$ - the mean total length of all ridges of the typical cell.
We can also define $\bar{\ell}_{X_{k}}$ and $\bar{\ell}_{X[\cdot]}$ in a similar way. For example,

$$
\begin{array}{ll}
\bar{\ell}_{\mathrm{E}[\text { hor }]}, \bar{\ell}_{\mathrm{E}[\mathrm{vert}]}- & \begin{array}{l}
\text { the mean length of the } \\
\text { and } \bar{\ell}_{\mathrm{E}[\pi]}
\end{array} \\
& \begin{array}{l}
\text { typical horizontal, vertical, } \\
\text { and } \pi \text {-edge, respectively, }
\end{array} \\
\bar{\ell}_{\mathrm{P}_{1}}, \bar{\ell}_{\mathrm{Z}_{1}} & -\begin{array}{l}
\text { the mean length of the } \\
\text { typical plate-side and the } \\
\text { typical ridge, respectively, }
\end{array} \\
\bar{\ell}_{\mathrm{Z}_{2}} & -\begin{array}{l}
\text { the mean perimeter of the } \\
\text { typical facet. }
\end{array}
\end{array}
$$

The notation above does not include, for instance, the mean total length of all edges of the typical cell. Therefore we use again the adjacency concept, analogous to the mean adjacencies $\mu_{\mathrm{XY}}$ :

$$
\begin{aligned}
\bar{\ell}_{X Y}-\quad \text { the mean total length of all } Y \text {-objects } \\
\text { adjacent to the typical } X \text {-object, where } \\
\\
\operatorname{dim}(Y \text {-object })=1 .
\end{aligned}
$$

For $\mathrm{X}=\mathrm{Z}$ and $\mathrm{Y}=\mathrm{E}$ we have

$$
\begin{aligned}
& \bar{\ell}_{Z E}-\text { the mean total length of all edges } \\
& \text { adjacent to the typical cell. }
\end{aligned}
$$

Some of these $\bar{\ell}_{\mathrm{XY}}$ mean values can be easily determined, for example

$$
\bar{\ell}_{\mathrm{PE}}=\bar{\ell}_{\mathrm{P}}, \quad \bar{\ell}_{\mathrm{Z}_{1} \mathrm{E}}=\bar{\ell}_{\mathrm{Z}_{1}}, \quad \bar{\ell}_{\mathrm{P}_{1} \mathrm{E}}=\bar{\ell}_{\mathrm{P}_{1}},
$$

but other examples (see Proposition 4.7) are more complicated and demonstrate the necessity of the notation.

Using the additional parameter $\bar{\gamma}_{E}$ of the planar tessellation $\mathscr{Y}^{\prime}$ - the length-weighted total $\rho$-intensity of the cells adjacent to the typical edge, we can calculate the mean values corresponding to the length measure of a column tessellation.

Theorem 4.6. Three mean values of primitive elements corresponding to the length measure of the column tessellation are given as follows:

$$
\begin{aligned}
& \bar{\ell}_{\mathrm{E}}=\frac{1}{2}\left(\frac{\bar{\gamma}_{\mathrm{E}}}{\bar{\alpha}_{\mathrm{E}}}+\frac{1}{\bar{\alpha}_{\mathrm{V}}}\right), \\
& \bar{\ell}_{\mathrm{P}}=\frac{\left(3 \bar{\gamma}_{\mathrm{E}}+2\right) \mu_{\mathrm{VE}}^{\prime}}{\left(\mu_{\mathrm{VE}}^{\prime}-2\right) \bar{\rho}_{\mathrm{Z}}+\mu_{\mathrm{VE}}^{\prime} \bar{\alpha}_{\mathrm{E}}}, \\
& \bar{\ell}_{\mathrm{Z}}=\frac{2\left(\mu_{\mathrm{VE}}^{\prime} \bar{\gamma}_{\mathrm{E}}+\mu_{\mathrm{VE}}^{\prime}-\phi\right)}{\left(\mu_{\mathrm{VE}}^{\prime}-2\right) \bar{\rho}_{\mathrm{Z}}} .
\end{aligned}
$$

Proof. 8. Recalling that a column tessellation has only horizontal and vertical edges,

$$
\lambda_{\mathrm{E}} \bar{\ell}_{\mathrm{E}}=\lambda_{\mathrm{E}[\text { hor }]} \bar{\ell}_{\mathrm{E}[\text { hor }]}+\lambda_{\mathrm{E}[\text { vert }]} \bar{\ell}_{\mathrm{E}[\text { vert }]}=\lambda_{\mathrm{E}}^{\prime} \bar{\gamma}_{\mathrm{E}}+\lambda_{\mathrm{V}}^{\prime} .
$$

With (ii) from Proposition 4.1 and the equation $\lambda_{\mathrm{E}}^{\prime} \bar{\alpha}_{\mathrm{E}}=\lambda_{V}^{\prime} \bar{\alpha}_{V}$ we obtain Eq. 8. 
9. Similarly, for the plates of $\mathscr{Y}$ we have

$$
\lambda_{\mathrm{P}} \bar{\ell}_{\mathrm{P}}=\lambda_{\mathrm{P}[\text { hor }]} \bar{\ell}_{\mathrm{P}[\text { hor }]}+\lambda_{\mathrm{P}[\text { vert }]} \bar{\ell}_{\mathrm{P}[\text { vert }]} .
$$

A cell z of the planar tessellation $\mathscr{Y}^{\prime}$ corresponds to $\rho_{z}$ horizontal plates of $\mathscr{Y}$ (they are vertical translations of z), hence $\lambda_{\mathrm{P}[\text { hor }]} \bar{\ell}_{\mathrm{P}[\text { hor }]}=\lambda_{\mathrm{Z}}^{\prime} \mathbb{E}_{\mathrm{Z}}^{\prime}\left(\ell^{\prime}(\mathrm{z}) \rho_{\mathrm{Z}}\right)=\lambda_{\mathrm{E}}^{\prime} \bar{\gamma}_{\mathrm{E}}$, from Remark 3.2. A vertical edge of $\mathscr{Y}$ on a line through a vertex $v$ of $\mathscr{Y}^{\prime}$ is adjacent to $m_{\mathrm{E}}^{\prime}(\mathrm{v})$ vertical plates. Furthermore any horizontal edge is adjacent to two vertical plates, see Property 3.5. Therefore, $\lambda_{\mathrm{P}[\text { vert }} \bar{\ell}_{\mathrm{P}[\text { vert }]}=\lambda_{\mathrm{V}}^{\prime} \mu_{\mathrm{VE}}^{\prime}+2 \lambda_{\mathrm{E}}^{\prime} \bar{\gamma}_{\mathrm{E}}=2 \lambda_{\mathrm{E}}^{\prime}+2 \lambda_{\mathrm{E}}^{\prime} \bar{\gamma}_{\mathrm{E}}$ and consequently

$$
\lambda_{\mathrm{P}} \bar{\ell}_{\mathrm{P}}=3 \lambda_{\mathrm{E}}^{\prime} \bar{\gamma}_{\mathrm{E}}+2 \lambda_{\mathrm{E}}^{\prime},
$$

which implies Eq. 9.

10. To determine the mean total length of all ridges of the typical cell, we use the fact that any horizontal ridge has multiplicity 2 (two cells with a common horizontal facet have horizontal ridges which are the sides of that facet). A point on a vertical line through a vertex $\vee$ of $\mathscr{Y}^{\prime}$ is an element of as many ridges as there are 0 -faces $z_{0}$ of $\mathscr{Y}^{\prime}$ which are located on $v$. That implies

$$
\lambda_{\mathrm{Z}} \bar{\ell}_{\mathrm{Z}}=2 \lambda_{\mathrm{P}[\mathrm{hor}]} \bar{\ell}_{\mathrm{P}[\mathrm{hor}]}+\lambda_{\mathrm{Z}_{0}}^{\prime}
$$

and we get Eq. 10 .

Other mean values corresponding to the length measure of the column tessellation can be computed in the same way by separating the roles of horizontal objects and vertical objects. For example,

- the mean length of the typical $\pi$-edge

$$
\bar{\ell}_{\mathrm{E}[\pi]}=\frac{\mu_{\mathrm{VE}}^{\prime} \bar{\gamma}_{\mathrm{E}}+2 \phi}{2\left(\bar{\alpha}_{V}+\phi \bar{\alpha}_{\mathrm{V}[\pi]}\right)},
$$

- the mean length of the typical ridge

$$
\bar{\ell}_{\mathrm{Z}_{1}}=\frac{1}{3 \bar{\beta}_{\mathrm{Z}_{0}}}\left(\frac{\mu_{\mathrm{VE}}^{\prime} \bar{\gamma}_{\mathrm{E}}}{\mu_{\mathrm{VE}}^{\prime}-\phi}+1\right)
$$

- the mean length of the typical plate-side

$$
\bar{\ell}_{\mathrm{P}_{1}}=\frac{\mu_{\mathrm{VE}}^{\prime}\left(3 \bar{\gamma}_{\mathrm{E}}+2\right)}{2\left(\mu_{\mathrm{VE}}^{\prime}-\phi\right) \bar{\beta}_{\mathrm{Z}_{0}}+4 \mu_{\mathrm{VE}}^{\prime} \bar{\alpha}_{\mathrm{E}}},
$$

- the mean perimeter of the typical facet

$$
\bar{\ell}_{Z_{2}}=\frac{2 \mu_{\mathrm{VE}}^{\prime} \bar{\gamma}_{\mathrm{E}}+2\left(\mu_{\mathrm{VE}}^{\prime}-\phi\right)}{\left(\mu_{\mathrm{VE}}^{\prime}-2\right) \bar{\rho}_{\mathrm{Z}}+\left(\mu_{\mathrm{VE}}^{\prime}-\phi\right) \bar{\beta}_{\mathrm{Z}_{0}}} .
$$

We also take care of results for some $\bar{\ell}_{\mathrm{XY}}$. It is interesting for us to calculate $\bar{\ell}_{\text {ZE }}$ and

$$
\begin{aligned}
& \bar{\ell}_{Z_{2} \mathrm{E}} \quad-\quad \text { the mean total length of all edges adjacent } \\
& \text { to the typical facet. }
\end{aligned}
$$

Proposition 4.7. The values of $\bar{\ell}_{\mathrm{ZE}}$ and $\bar{\ell}_{\mathrm{Z}_{2} \mathrm{E}}$ are given as follows:

$$
\begin{aligned}
\bar{\ell}_{\mathrm{ZE}} & =\frac{\mu_{\mathrm{VE}}^{\prime}\left(3 \bar{\gamma}_{\mathrm{E}}+2\right)}{\left(\mu_{\mathrm{VE}}^{\prime}-2\right) \bar{\rho}_{\mathrm{Z}}}, \\
\bar{\ell}_{\mathrm{Z}_{2} \mathrm{E}} & =\frac{5 \mu_{\mathrm{VE}}^{\prime} \bar{\gamma}_{\mathrm{E}}+4 \mu_{\mathrm{VE}}^{\prime}-2 \phi}{2\left[\left(\mu_{\mathrm{VE}}^{\prime}-2\right) \bar{\rho}_{\mathrm{Z}}+\left(\mu_{\mathrm{VE}}^{\prime}-\phi\right) \bar{\beta}_{\mathrm{Z}_{0}}\right]} .
\end{aligned}
$$

Proof. Based on the properties of the column tessellation, it is not difficult to see that

$$
\begin{aligned}
\lambda_{\mathrm{Z}} \bar{\ell}_{\mathrm{ZE}} & =\lambda_{\mathrm{Z}} \bar{\ell}_{\mathrm{ZE}[\mathrm{hor}]}+\lambda_{\mathrm{Z}} \bar{\ell}_{\mathrm{ZE}[\mathrm{vert}]} \\
& =3 \lambda_{\mathrm{E}[\mathrm{hor}]} \bar{\ell}_{\mathrm{E}[\mathrm{hor}]}+\lambda_{\mathrm{Z}}^{\prime} \mu_{\mathrm{ZV}}^{\prime} \\
& =3 \lambda_{\mathrm{E}}^{\prime} \bar{\gamma}_{\mathrm{E}}+2 \lambda_{\mathrm{E}}^{\prime} .
\end{aligned}
$$

To prove the second formula for $\bar{\ell}_{\mathrm{Z}_{2} \mathrm{E}}$, firstly we note that any horizontal edge of $\mathscr{Y}$ is contained in the boundary of two horizontal and two vertical cell-facets and in the interior of a further vertical cell-facet. This explains the summand $5 \lambda_{\mathrm{E}}^{\prime} \bar{\gamma}_{\mathrm{E}}$. Secondly, a vertical edge of $\mathscr{Y}$ which is corresponding to a non $\pi$-vertex $\vee$ of $\mathscr{Y}^{\prime}$ is adjacent to $2 m_{\mathrm{E}}^{\prime}(\mathrm{v})=2 m_{\mathrm{Z}_{0}}^{\prime}(\mathrm{v})$ vertical cell facets, whereas a vertical edge corresponding to a $\pi$-vertex $v$ is adjacent to $2 m_{\mathrm{E}}^{\prime}(\mathrm{v})-1=2 m_{\mathrm{Z}_{0}}^{\prime}(\mathrm{v})+1$ facets. This creates the two summands $2 \lambda_{\mathrm{Z}_{0}}^{\prime}+\lambda_{\mathrm{V}[\pi]}^{\prime}=\lambda_{\mathrm{Z}_{0}}^{\prime}+2 \lambda_{\mathrm{E}}^{\prime}$. We obtain

$$
\begin{aligned}
\lambda_{\mathrm{Z}_{2}} \bar{\ell}_{\mathrm{Z}_{2} \mathrm{E}} & =5 \lambda_{\mathrm{E}}^{\prime} \bar{\gamma}_{\mathrm{E}}+2 \lambda_{\mathrm{E}}^{\prime}+\lambda_{\mathrm{Z}_{0}}^{\prime} \\
& =5 \lambda_{\mathrm{E}}^{\prime} \bar{\gamma}_{\mathrm{E}}+\lambda_{\mathrm{Z}}^{\prime} \frac{4 \mu_{\mathrm{VE}}^{\prime}-2 \phi}{\mu_{\mathrm{VE}}^{\prime}-2},
\end{aligned}
$$

which completes our proof.

For the mean values corresponding to the length measure of column tessellations of constant cell-height 1 , we have (using the metric parameter $\bar{\ell}_{\mathrm{E}}^{\prime}$ from the planar tessellation)

$$
\begin{aligned}
& \bar{\ell}_{\mathrm{E}}=\frac{1}{2}\left(\bar{\ell}_{\mathrm{E}}^{\prime}+\frac{1}{\mu_{\mathrm{VE}}^{\prime}}\right), \quad \bar{\ell}_{\mathrm{E}[\pi]}=\frac{2\left(\mu_{\mathrm{VE}}^{\prime} \bar{\ell}_{\mathrm{E}}^{\prime}+\phi\right)}{\mu_{\mathrm{VE}}^{\prime}\left(2+\mu_{\mathrm{EV}[\pi]}^{\prime}\right)}, \\
& \bar{\ell}_{\mathrm{P}}=\frac{2 \mu_{\mathrm{VE}}^{\prime}\left(3 \bar{\ell}_{\mathrm{E}}^{\prime}+1\right)}{3 \mu_{\mathrm{VE}}^{\prime}-2}, \quad \bar{\ell}_{\mathrm{P}_{1}}=\frac{\mu_{\mathrm{VE}}^{\prime}\left(3 \bar{\ell}_{\mathrm{E}}^{\prime}+1\right)}{5 \mu_{\mathrm{VE}}^{\prime}-\phi},
\end{aligned}
$$




$$
\begin{aligned}
\bar{\ell}_{\mathrm{Z}} & =\frac{2\left(2 \mu_{\mathrm{VE}}^{\prime} \bar{\ell}_{\mathrm{E}}^{\prime}+\mu_{\mathrm{VE}}^{\prime}-\phi\right)}{\mu_{\mathrm{VE}}^{\prime}-2}, \\
\bar{\ell}_{\mathrm{Z}_{1}} & =\frac{1}{3}+\frac{2 \mu_{\mathrm{VE}}^{\prime} \bar{\ell}_{\mathrm{E}}^{\prime}}{3\left(\mu_{\mathrm{VE}}^{\prime}-\phi\right)}, \\
\bar{\ell}_{\mathrm{Z}_{2}} & =\frac{2\left(2 \mu_{\mathrm{VE}}^{\prime} \bar{\ell}_{\mathrm{E}}^{\prime}+\mu_{\mathrm{VE}}^{\prime}-\phi\right)}{2 \mu_{\mathrm{VE}}^{\prime}-\phi-2}, \\
\bar{\ell}_{\mathrm{Z}_{2} \mathrm{E}} & =\frac{5 \mu_{\mathrm{VE}}^{\prime} \bar{\ell}_{\mathrm{E}}^{\prime}+2 \mu_{\mathrm{VE}}^{\prime}-\phi}{2 \mu_{\mathrm{VE}}^{\prime}-\phi-2}, \quad \bar{\ell}_{\mathrm{ZE}}=\frac{2 \mu_{\mathrm{VE}}^{\prime}\left(3 \bar{\ell}_{\mathrm{E}}^{\prime}+1\right)}{\mu_{\mathrm{VE}}^{\prime}-2} .
\end{aligned}
$$

Metric formulae involving areas and volumes: Firstly we deal with areas, using analogous notation. Later in this sub-section, we consider volumes.

$\bar{a}_{\mathrm{X}} \quad-\quad$ the mean total area of all 2-faces of the typical X-object, where $\operatorname{dim}(X$-object $) \geq 2$.

In this case we have

$\bar{a}_{\mathrm{P}} \quad-\quad$ the mean area of the typical plate,

$\bar{a}_{Z} \quad-\quad$ the mean surface area of the typical cell, and,

$\bar{a}_{Z_{2}} \quad-$ the mean area of the typical cell-facet.

To determine these mean values for the column tessellation $\mathscr{Y}$, we use two additional mean values of the planar tessellation $\mathscr{Y}^{\prime}$, namely $\bar{\gamma}_{Z}$ and $\bar{\ell}_{\mathrm{E}}^{\prime}$.

Theorem 4.8. The mean area of the typical plate of the column tessellation is

$$
\bar{a}_{\mathrm{P}}=\frac{\left(\mu_{\mathrm{VE}}^{\prime}-2\right) \bar{\gamma}_{\mathrm{Z}}+\mu_{\mathrm{VE}}^{\prime} \bar{\ell}_{\mathrm{E}}^{\prime}}{\left(\mu_{\mathrm{VE}}^{\prime}-2\right) \bar{\rho}_{\mathrm{Z}}+\mu_{\mathrm{VE}}^{\prime} \bar{\alpha}_{\mathrm{E}}} .
$$

Proof. For a cell z of $\mathscr{Y}^{\prime}$ the corresponding column contains on its boundary the vertical plates of $\mathscr{Y}$. These plates are rectangles with two horizontal edges corresponding to an edge of $\mathscr{Y}^{\prime}$ adjacent to $\mathrm{z}$, that is, $\lambda_{\mathrm{P}[\text { vert }]} \bar{a}_{\mathrm{P}[\text { vert }]}=\lambda_{\mathrm{E}}^{\prime} \bar{\ell}_{\mathrm{E}}^{\prime}$. Because all horizontal plates of $\mathscr{Y}$ are translations of the cells of $\mathscr{Y}^{\prime}$, we have $\lambda_{\mathrm{P}[\text { hor }]} \bar{a}_{\mathrm{P}[\text { hor }]}=\lambda_{\mathrm{Z}}^{\prime} \mathbb{E}_{\mathrm{Z}}^{\prime}\left(a^{\prime}(\mathrm{z}) \rho_{\mathrm{Z}}\right)=\lambda_{\mathrm{Z}}^{\prime} \bar{\gamma}_{\mathrm{Z}}$; see Remark 3.2. Therefore

$\lambda_{\mathrm{P}} \bar{a}_{\mathrm{P}}=\lambda_{\mathrm{P}[\text { hor }]} \bar{a}_{\mathrm{P}[\text { hor }]}+\lambda_{\mathrm{P}[\text { vert }]} \bar{a}_{\mathrm{P}[\text { vert }]}=\lambda_{\mathrm{Z}}^{\prime} \bar{\gamma}_{\mathrm{Z}}+\lambda_{\mathrm{E}}^{\prime} \bar{\ell}_{\mathrm{E}}^{\prime}$,

which implies our result.

Using the fact that each plate of a spatial tessellation always belongs to two cells and two facets, we have $\lambda_{\mathrm{Z}} \bar{a}_{\mathrm{Z}}=2 \lambda_{\mathrm{P}} \bar{a}_{\mathrm{P}}$ and $\lambda_{\mathrm{Z}_{2}} \bar{a}_{\mathrm{Z}_{2}}=2 \lambda_{\mathrm{P}} \bar{a}_{\mathrm{P}}$. With the help of Proposition 4.1 we infer that

$$
\begin{aligned}
\bar{a}_{\mathrm{Z}} & =\frac{2}{\bar{\rho}_{\mathrm{Z}}}\left(\bar{\gamma}_{\mathrm{Z}}+\frac{\mu_{\mathrm{VE}}^{\prime} \bar{\ell}_{\mathrm{E}}^{\prime}}{\mu_{\mathrm{VE}}^{\prime}-2}\right), \\
\bar{a}_{\mathrm{Z}_{2}} & =\frac{\left(\mu_{\mathrm{VE}}^{\prime}-2\right) \bar{\gamma}_{\mathrm{Z}}+\mu_{\mathrm{VE}}^{\prime} \bar{\ell}_{\mathrm{E}}^{\prime}}{\left(\mu_{\mathrm{VE}}^{\prime}-2\right) \bar{\rho}_{\mathrm{Z}}+\left(\mu_{\mathrm{VE}}^{\prime}-\phi\right) \bar{\beta}_{\mathrm{Z}_{0}}} .
\end{aligned}
$$

Also for the area measure we can consider mean values of type $\bar{a}_{X Y}-$ the mean total area of all Y-type objects adjacent to the typical $X$-object. Again some equations are obvious:

$$
\bar{a}_{\mathrm{ZP}}=\bar{a}_{\mathrm{Z}}, \quad \bar{a}_{\mathrm{Z}_{2} \mathrm{P}}=\bar{a}_{\mathrm{Z}_{2}} .
$$

It is interesting in this context to evaluate the mean total area of all facets adjacent to the typical cell, namely $\bar{a}_{Z Z_{2}}$. In a facet-to-facet spatial tessellation, it is easy to see that $\bar{a}_{Z Z_{2}}=2 \bar{a}_{Z}$, because each cell-facet is a plate and the class $Z_{2}$ of cell-facets is equal to the class $P$ of plates up to the multiplicity 2 . It is difficult to determine $\bar{a}_{\mathrm{ZZ}}$ for an arbitrary non-facet-to-facet spatial tessellation; we only know that $\bar{a}_{\mathrm{ZZ}} \geq \bar{a}_{\mathrm{Z}}$. For a column tessellation, however, we can compute $\bar{a}_{Z Z_{2}}$, using the fact that each horizontal facet of a cell is also a facet of one another cell and each vertical facet is an element of $Z_{2}$ with multiplicity 1 . So any cell $z$ of $\mathscr{Y}$ is adjacent to its facets, obviously, and to the two horizontal facets of the neighbouring cells within the same column. Thes are identical to the base and top facet of $z$. There are no further facets adjacent to $z$. Therefore we obtain

$$
\begin{aligned}
\lambda_{\mathrm{Z}} \bar{a}_{\mathrm{ZZ} 2} & =\lambda_{\mathrm{Z}} \bar{a}_{\mathrm{Z}}+2 \lambda_{\mathrm{P}[\mathrm{hor}]} \bar{a}_{\mathrm{P}[\mathrm{hor}]} \\
& =\lambda_{\mathrm{Z}}^{\prime}\left(4 \bar{\gamma}_{\mathrm{Z}}+2 \frac{\mu_{\mathrm{VE}}^{\prime} \bar{\ell}_{\mathrm{E}}^{\prime}}{\mu_{\mathrm{VE}}^{\prime}-2}\right),
\end{aligned}
$$

hence

$$
\bar{a}_{\mathrm{ZZ}}=\frac{2}{\bar{\rho}_{\mathrm{Z}}}\left(2 \bar{\gamma}_{\mathrm{Z}}+\frac{\mu_{\mathrm{VE}}^{\prime} \bar{\ell}_{\mathrm{E}}^{\prime}}{\mu_{\mathrm{VE}}^{\prime}-2}\right) .
$$

Finally, we present results involving volumes.

Theorem 4.9. The mean volume of the typical cell of the column tessellation, denoted by $\bar{v}_{\mathrm{Z}}$, is

$$
\bar{v}_{\mathrm{Z}}=\frac{1}{\lambda_{\mathrm{Z}}^{\prime} \bar{\rho}_{\mathrm{Z}}} \text {. }
$$

Proof. It is obvious from the fact that $\lambda_{\mathrm{Z}} \bar{v}_{\mathrm{Z}}=1$.

The corresponding area and volume mean values of column tessellations of constant cell-height 1 are

$$
\begin{aligned}
\bar{a}_{\mathrm{P}} & =\frac{\left(\mu_{\mathrm{VE}}^{\prime}-2\right) \bar{a}_{\mathrm{Z}}^{\prime}+\mu_{\mathrm{VE}}^{\prime} \bar{\ell}_{\mathrm{E}}^{\prime}}{3 \mu_{\mathrm{VE}}^{\prime}-2}, \\
\bar{a}_{\mathrm{Z}} & =2\left(\bar{a}_{\mathrm{Z}}^{\prime}+\frac{\mu_{\mathrm{VE}}^{\prime} \bar{\ell}_{\mathrm{E}}^{\prime}}{\mu_{\mathrm{VE}}^{\prime}-2}\right), \\
\bar{a}_{\mathrm{Z}} & =\frac{\left(\mu_{\mathrm{VE}}^{\prime}-2\right) \bar{a}_{\mathrm{Z}}^{\prime}+\mu_{\mathrm{VE}}^{\prime} \bar{\ell}_{\mathrm{E}}^{\prime}}{2 \mu_{\mathrm{VE}}^{\prime}-\phi-2}, \\
\bar{a}_{\mathrm{ZZ}} & =2\left(2 \bar{a}_{\mathrm{Z}}^{\prime}+\frac{\mu_{\mathrm{VE}}^{\prime} \bar{\ell}_{\mathrm{E}}^{\prime}}{\mu_{\mathrm{VE}}^{\prime}-2}\right), \\
\bar{v}_{\mathrm{Z}} & =\frac{1}{\lambda_{\mathrm{Z}}^{\prime}} .
\end{aligned}
$$




\section{THREE EXAMPLES}

Concluding the paper we will give three examples for column tessellations. The generating planar tessellations are the Poisson line tessellation (PLT), the STIT tessellation and the Poisson- Voronoi tessellation (PVT), respectively. We will consider the column tessellations with constant cell height $1\left(\rho_{Z}=1\right)$ and we restrict for the planar mosaics to the stationary and isotropic case. The intensities, topological/ interior parameters and metric mean values for those column tessellations are presented in Table 2. To facilitate the comparability of the results we assume that all the underlying planar tessellations have the same cellintensity $\lambda_{\mathrm{Z}}^{\prime}$. In Table 1 the seven necessary parameters of the planar PLT, STIT and PVT are given (Chiu et al., 2013; Nagel and Weiss, 2008). In a PLT all vertices have 4 emanating edges $\left(\mu_{\mathrm{VE}}^{\prime}=4, \mu_{\mathrm{VE}}^{\prime(2)}=\right.$ 16), whereas in STIT and PVT all vertices have 3 emanating edges $\left(\mu_{\mathrm{VE}}^{\prime}=3, \mu_{\mathrm{VE}}^{\prime(2)}=9\right)$. PLT and PVT are side-to-side $\left(\phi=\mu_{\mathrm{EV}[\pi]}^{\prime}=0\right)$; a STIT tessellation is non side-to-side and all vertices are $\pi$-vertices $(\phi=1$, $\mu_{\mathrm{EV}[\pi]}^{\prime}=2$ ). Through the paper all our results were considered for the special case of a column tessellation with constant height 1 . In Remark 3.3 the $\alpha-\beta$ and $\gamma$-mean values are given, Remark 4.3 presents the intensities and topological/interior mean values and the metric mean values are given in the previous section. Using those results the entries in Table 2 and other interesting quantities of those column tessellations can be computed.

Table 1. Seven parameters of the planar tessellation.

\begin{tabular}{lccc}
\hline $\mathscr{Y}^{\prime}$ & PLT & STIT & PVT \\
\hline$\lambda_{\mathrm{V}}^{\prime}$ & $\lambda_{\mathrm{Z}}^{\prime}$ & $2 \lambda_{\mathrm{Z}}^{\prime}$ & $2 \lambda_{\mathrm{Z}}^{\prime}$ \\
$\mu_{\mathrm{VE}}^{\prime}$ & 4 & 3 & 3 \\
$\mu_{\mathrm{VE}}^{\prime(2)}$ & 16 & 9 & 9 \\
$\phi$ & 0 & 1 & 0 \\
$\mu_{\mathrm{EV}[\pi]}^{\prime}$ & 0 & 2 & 0 \\
$\bar{\ell}_{\mathrm{E}}^{\prime}$ & $\frac{\sqrt{\pi}}{2 \sqrt{\lambda_{\mathrm{Z}}^{\prime}}}$ & $\frac{\sqrt{\pi}}{3 \sqrt{\lambda_{\mathrm{Z}}^{\prime}}}$ & $\frac{2}{3 \sqrt{\lambda_{\mathrm{Z}}^{\prime}}}$ \\
$\bar{a}_{\mathrm{Z}}^{\prime}$ & $\frac{1}{\lambda_{\mathrm{Z}}^{\prime}}$ & $\frac{1}{\lambda_{\mathrm{Z}}^{\prime}}$ & $\frac{1}{\lambda_{\mathrm{Z}}^{\prime}}$ \\
\hline
\end{tabular}

Table 2. Fifteen mean values of the corresponding column tessellation with height 1.

\begin{tabular}{|c|c|c|c|}
\hline $\mathscr{Y} / \mathscr{Y}^{\prime}$ & PLT & STIT & PVT \\
\hline$\lambda_{V}$ & $4 \lambda_{z}^{\prime}$ & $6 \lambda_{z}^{\prime}$ & $6 \lambda_{z}^{\prime}$ \\
\hline$\lambda_{P}$ & $5 \lambda_{Z}^{\prime}$ & $7 \lambda_{Z}^{\prime}$ & $7 \lambda_{z}^{\prime}$ \\
\hline$\mu_{\mathrm{PV}}$ & $\frac{28}{5}$ & $\frac{36}{7}$ & $\frac{36}{7}$ \\
\hline$\mu_{\mathrm{EP}}$ & $\frac{7}{2}$ & 3 & 3 \\
\hline$\xi$ & $\frac{1}{2}$ & 1 & $\frac{1}{2}$ \\
\hline$\kappa$ & 0 & $\frac{2}{3}$ & 0 \\
\hline$\psi$ & 3 & 2 & 2 \\
\hline$\tau$ & 2 & $\frac{4}{3}$ & 1 \\
\hline $\bar{\ell}_{\mathrm{E}}$ & $\frac{\sqrt{\pi}}{4 \sqrt{\lambda_{\mathrm{Z}}^{\prime}}}+\frac{1}{8}$ & $\frac{\sqrt{\pi}}{6 \sqrt{\lambda_{\mathrm{Z}}^{\prime}}}+\frac{1}{6}$ & $\frac{1}{3 \sqrt{\lambda_{\mathrm{Z}}^{\prime}}}+\frac{1}{6}$ \\
\hline $\bar{\ell}_{P}$ & $\frac{6 \sqrt{\pi}}{5 \sqrt{\lambda_{\mathrm{Z}}^{\prime}}}+\frac{4}{5}$ & $\frac{6 \sqrt{\pi}}{7 \sqrt{\lambda_{\mathrm{Z}}^{\prime}}}+\frac{6}{7}$ & $\frac{12}{7 \sqrt{\lambda_{\mathrm{Z}}^{\prime}}}+\frac{6}{7}$ \\
\hline $\bar{\ell}_{\mathrm{z}}$ & $\frac{4 \sqrt{\pi}}{\sqrt{\lambda_{Z}^{\prime}}}+4$ & $\frac{4 \sqrt{\pi}}{\sqrt{\lambda_{Z}^{\prime}}}+4$ & $\frac{8}{\sqrt{\lambda_{Z}^{\prime}}}+6$ \\
\hline $\bar{\ell}_{\text {ZE }}$ & $\frac{6 \sqrt{\pi}}{\sqrt{\lambda_{Z}^{\prime}}}+4$ & $\frac{6 \sqrt{\pi}}{\sqrt{\lambda_{Z}^{\prime}}}+6$ & $\frac{12}{\sqrt{\lambda_{Z}^{\prime}}}+6$ \\
\hline $\bar{\ell}_{\mathrm{Z}_{2} \mathrm{E}}$ & $\frac{5 \sqrt{\pi}}{3 \sqrt{\lambda_{Z}^{\prime}}}+\frac{4}{3}$ & $\frac{5 \sqrt{\pi}}{3 \sqrt{\lambda_{Z}^{\prime}}}+\frac{5}{3}$ & $\frac{5}{2 \sqrt{\lambda_{Z}^{\prime}}}+\frac{3}{2}$ \\
\hline $\bar{a}_{\mathrm{P}}$ & $\frac{1}{5 \lambda_{\mathrm{Z}}^{\prime}}+\frac{\sqrt{\pi}}{5 \sqrt{\lambda_{\mathrm{Z}}^{\prime}}}$ & $\frac{1}{7 \lambda_{Z}^{\prime}}+\frac{\sqrt{\pi}}{7 \sqrt{\lambda_{Z}^{\prime}}}$ & $\frac{1}{7 \lambda_{Z}^{\prime}}+\frac{2}{7 \sqrt{\lambda_{Z}^{\prime}}}$ \\
\hline $\bar{a}_{\mathrm{ZZ}}$ & $\frac{4}{\lambda_{\mathrm{Z}}^{\prime}}+\frac{2 \sqrt{\pi}}{\sqrt{\lambda_{\mathrm{Z}}^{\prime}}}$ & $\frac{4}{\lambda_{\mathrm{Z}}^{\prime}}+\frac{2 \sqrt{\pi}}{\sqrt{\lambda_{\mathrm{Z}}^{\prime}}}$ & $\frac{4}{\lambda_{\mathrm{Z}}^{\prime}}+\frac{4}{\sqrt{\lambda_{\mathrm{Z}}^{\prime}}}$ \\
\hline
\end{tabular}

\section{ACKNOWLEDGEMENT}

We thank the two referees for their helpful comments. The first two authors were supported by the German research foundation (DFG), grant WE 1799/3-1.

\section{REFERENCES}

Chiu SN, Stoyan D, Kendall WS, Mecke J (2013). Stochastic geometry and its applications. 3rd Ed. Chichester: Wiley.

Cowan R (2013). Line segments in the isotropic planar STIT tessellation. Adv Appl Probab 45:295-311.

Cowan R, Thäle C (2014). The character of planar tessellations which are not side-to-side. Image Anal Stereol 33:39-54. 
Cowan R, Weiss V (2015). Constraints on the fundamental topological parameters of spatial tessellations. Math Nachr 288:540-65.

Grünbaum B, Shephard GC (1987). Tilings and Patterns. New York: WH Freeman.

Mecke J (1984). Parametric representation of mean values for stationary random mosaics. Math Operationsforsch Statist Ser Statist 15:437-42.

Mecke J, Nagel W, Weiss V (2008). The iteration of random tessellations and a construction of a homogeneous process of cell divisions. Adv Appl Probab 40:49-59.

Møller J (1989). Random tessellations in $\mathbb{R}^{d}$. Adv Appl Probab 21:37-73.

Mosser LJ, Matthäi SK (2014). Tessellations stable under iteration - Evaluation of application as an improved stochastic discrete fracture modeling algorithm. Proc Int Discrete Fracture Network Eng Conf. Oct 19-22. Vancouver.

Nagel W, Weiss V (2005). Crack STIT tessellations
- characterization of stationary random tessellations stable with respect to iteration. Adv Appl Probab 37:859-83.

Nagel W, Weiss V (2008). Mean values for homogeneous STIT tessellations in 3D. Image Anal Stereol 27:29-37.

Radecke W (1980). Some mean-value relations on stationary random mosaics in the space. Math Nachr 97:203-10.

Schneider R, Weil W (2008). Stochastic and integral geometry. Berlin: Springer.

Thäle C, Weiss V (2013). The combinatorial structure of spatial STIT tessellations. Discrete Comput Geom 50:649-72.

Thäle C, Weiss V, Nagel W (2012). Spatial STIT tessellations: Distributional results for I-segments. Adv Appl Probab 44:1-20.

Weiss V, Cowan R (2011). Topological relationships in spatial tessellations. Adv Appl Probab 43:963-84. 